Juan Garrido, Francisco Vázquez and Fernando Morilla, Multivariable PID control by decoupling, International Journal of Systems Science, volume 47, number 5, pp. 1054-1072, 2016,

ISSN 0020-7721, The Version of Record of this manuscript has been published and is available in http://www.tandfonline.com/doi/abs/10.1080/00207721.2014.911390

(http://dx.doi.org/10.1080/00207721.2014.911390.)

\title{
MULTIVARIABLE PID CONTROL BY DECOUPLING
}

Juan Garrido†*, Francisco Vázquez†, Fernando Morillał

† Department of Computer Science and Numerical Analysis, University of Córdoba, Campus de Rabanales, 14071, Córdoba, Spain

*Fax: (+34)957218729; e-mail: juan.garrido@uco.es

$¥$ Department of Computer Science and Automatic Control, UNED, Juan del Rosal 16, 28040, Madrid, Spain Department, University, City, Country

\section{Abstract}

This paper presents a new methodology to design multivariable PID controllers based on decoupling control. The method is presented for general $n \times n$ processes. In the design procedure, an ideal decoupling control with integral action is designed to minimize interactions. It depends on the desired open loop processes that are specified according to realizability conditions and desired closed loop performance specifications. These realizability conditions are stated and three common cases to define the open loop processes are studied and proposed. Then, controller elements are approximated to PID structure. From a practical point of view, the windup problem is also considered and a new anti-windup scheme for multivariable PID controller is proposed. Comparisons with other works demonstrate the effectiveness of the methodology through the use of several simulation examples and an experimental lab process.

Keywords: decoupling control; PID control; multivariable control; centralized control.

\section{Introduction}

Most industrial processes consist of multiple input and output signals, and there are 
often complicated couplings between them, which may cause difficulties in feedback controller design. To cope with this problem, control engineers traditionally use singleloop PID controllers because they are easily understood and implemented (Shinskey, 1979). These decentralized approaches can work properly when the interactions in different channels of the process are modest (Chien, Huang, \& Yang, 1999; Lee, Lee, Kim, \& Lee, 2004; Vázquez, Morilla, \& Dormido, 1999; Qiang Xiong \& Cai, 2006). However, when interactions are important, the decoupling is often treated inefficiently, e.g., by detuning control loops. Consequently, the decoupling performance obtained from well-established single-loop PID tuning techniques is not satisfactory. In fact, some leading controller manufacturers consider this poor decoupling of multivariable processes as one of the main control problems in the industry (Wang, 2003). In these cases, a full matrix controller (centralized control) is advised.

However, although centralized control can reduce the interactions and improve the performance of the multivariable control system, it is important to point out that this approach shows some disadvantages over decentralized control:

- From a theoretical point of view, the centralized control methodologies are more complicated to carry out than decentralized control methods.

- Centralized controllers can be more sensitive to modeling errors and uncertainties than decentralized controllers, specifically in ill-conditioned processes.

- Decentralized control usually achieves better disturbance rejection than centralized control. Thus, sometimes two degree of freedom control structures are convenient when a trade-off between tracking references and disturbance rejections is necessary.

- Transmission zeros of the controller are added to those of the process with the corresponding closed-loop performance limitations in case of RHP transmission zeros. 
- From an industrial point of view, important practical issues as anti-windup mechanism or bumpless transfer between manual and automatic mode are generally more difficult to implement.

Therefore, the centralized control scheme should be implemented when it is justified according to the performance specifications and the process characteristics.

In the literature, centralized control design is usually approached in two different ways: a decoupling network combined with a diagonal decentralized controller, or a pure centralized strategy. Figure 1a represents the general control scheme of the first approach, where $\mathrm{G}(\mathrm{s}), \mathrm{D}(\mathrm{s})$ and $\mathrm{C}(\mathrm{s})$ are the process matrix, the decoupling matrix and the decentralized control matrix, respectively. The decoupler is used to minimize the process interaction in such a way that the control matrix $\mathrm{C}(\mathrm{s})$ sees the apparent process $\mathrm{G}(\mathrm{s}) \cdot \mathrm{D}(\mathrm{s})$ as a set of completely independent processes (Cai, Ni, He, \& Ni, 2008; Garrido, Vázquez, \& Morilla, 2011, 2012; Garrido, Vázquez, Morilla, \& Hägglund, 2011; Jevtovic \& Matausek, 2010; Rajapandiyan \& Chidambaram, 2012; Shen, Sun, \& Li, 2012; Waller, Waller, \& Waller, 2003). Then, the controllers are designed using some single-loop methodology.

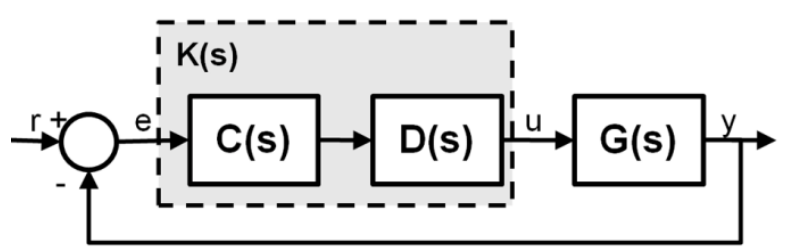

(a)

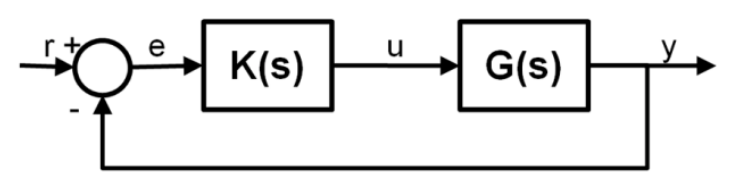

(b)

Figure 1. Centralized control schemes: (a) decoupling control system, (b) purely centralized control system. 
On the other hand, Figure 1b shows a pure centralized control system with K(s) being the n-dimensional full matrix controller. $\mathrm{K}(\mathrm{s})$ works as the only block to control the different measurement signals and to reduce the interactions. Under the paradigm of decoupling control, some methodologies have been developed using this approach (Lieslehto, 1996; Liu, Zhang, \& Gao, 2007; Wang, Zhang, \& Chiu, 2002, 2003; Wang, Zou, Lee, \& Bi, 1997; Q. Xiong, Cai, \& He, 2007; Zhang, Chen, \& Ou, 2006). Most of them propose to find a $\mathrm{K}(\mathrm{s})$ in such a way that the closed loop transfer matrix $H(s)=G(s) \cdot K(s) \cdot[I+G(s) \cdot K(s)]^{-1}$ is decoupled over some desired bandwidth. This goal is achieved if the open loop transfer matrix $\mathrm{L}(\mathrm{s})=\mathrm{G}(\mathrm{s}) \cdot \mathrm{K}(\mathrm{s})$ is diagonal over that bandwidth. Hence, the decoupling control techniques, such as the proposed one in this work, are very similar to the decoupler design methodologies (Garrido et al., 2012; Morilla, Garrido, \& Vázquez, 2013).

The complexity of the resultant controller elements of K(s) can be very different depending on the methodology. For instance, in (Wang et al., 2003), a full-dimensional non-PID is obtained from a recursive least square optimization problem. Other authors propose an analytical decoupling control strategy on the basis of the $\mathrm{H}_{2}$ optimal performance specifications (Liu et al., 2007). Nevertheless, PID controllers have dominated applications for more than 70 years. They are preferred over more advanced controllers in practical applications unless PID controllers cannot meet the specifications. Therefore, several methodologies (Lieslehto, 1996; Q. Xiong et al., 2007) obtain a multivariable PID controller as resultant controller K(s). One of the conclusions reached at the IFAC Conference on Advances in PID Control, held in Brescia in 2012, was that PID control will stay as the preferred control algorithm at the bottom layer in spite of other promising proposals such as MPC paradigms (Alcántara, Vilanova, \& Pedret, 2013). One the other hand, model predictive control (MPC) is 
becoming the standard solution to multivariable control problems in the process industry, and new developments seem to make it suitable at lower levels (Zhu, Patwardhan, Wagner, \& Zhao, 2013). Therefore, the trend in the near future should be directed towards good collaboration between both technologies instead of competition (Darby \& Nikolaou, 2012). Hence, new multivariable PID design methods are interesting for the process industry. This design can be approached from state space methods that usually try to minimize a $\mathrm{H} \infty$ norm as performance measure (Ochi \& Yokoyama, 2012; Saeki, 2006; Zheng, Wang, \& Lee, 2002). However, these procedures mainly involve delay-free systems, and many industrial processes contain time delays (Zhang et al., 2006). Thus, a transfer matrix approach has been preferred in this work.

Most of the previous centralized methodologies use the conventional scheme of Figure $1 \mathrm{~b}$ in which there is a direct relationship between the controller elements to be implemented and the elements of $\mathrm{K}(\mathrm{s})$. The process inputs $\mathrm{u}$ are derived by a timeweighted combination of the error signals e. In this case, after specifying a desired diagonal matrix $\mathrm{L}(\mathrm{s})$ or $\mathrm{H}(\mathrm{s})$ as requirement in the design, the matrix $\mathrm{K}(\mathrm{s})$ can be calculated according to (1) or (2), respectively.

$$
\begin{gathered}
K(s)=G^{-1}(s) \cdot L(s) \\
K(s)=G^{-1}(s) \cdot\left(H^{-1}(s)-I\right)^{-1}
\end{gathered}
$$

The main problem of this procedure is the fact that the complexity of controller elements tends to increase for high-dimensional multivariable systems, which may require model reductions and approximations. An alternative method, called centralized inverted decoupling control, maintains very simple controller elements and open loop processes independently of the system size (Garrido, Vázquez, \& Morilla, 2013). Moreover, it presents several practical advantages on implementation. Nevertheless, the 
method also has an important disadvantage: because of stability problems, it cannot be applied to processes with multivariable right half plane (RHP) zeros, that is, RHP zeros in the determinant of the process transfer matrix G(s). In this case, the conventional decoupling scheme would be required despite its disadvantages.

This work proposes a new centralized PID control methodology which is based in the scheme of conventional centralized decoupling control (Figure 1b) and which is performed focusing on stable processes with possibly RHP zeros and time delays. An initial version of this methodology was introduced for $2 \times 2$ processes in (Morilla, Vázquez, \& Garrido, 2008). In this work, its formulation was developed and generalized to $n \times n$ processes while the realizability conditions were presented. Finally, a centralized PID control is obtained by controller reduction for implementation.

The paper is structured as follows. Section 2 presents the general formulation of the methodology for $n \times n$ systems. The aspects related to realizability conditions and performance specifications are also discussed. Section 3 describes some design and practical considerations related to the proposed methodology, such as the approximation method, the reduction to multivariable PID control and a new anti-windup implementation scheme for multivariable PID controllers. In Section 4, the performance of the proposed method is tested and compared with other techniques using several simulation examples and a real quadruple tank process. Finally, conclusions are summarized in Section 5.

\section{Methodology}

Given a multivariable process $\mathrm{G}(\mathrm{s})$ and following the centralized control system depicted in Figure 1b, it is possible to calculate the controller matrix K(s) from (1) after specifying a diagonal open loop process $\mathrm{L}(\mathrm{s})$. It is assumed that the process $\mathrm{G}(\mathrm{s})$ may have RHP zeros and multiple time delays, but it does not have unstable poles in the left 
half plane. The expression of the entire controller matrix K(s) is obtained as follows:

$$
K=G^{-1} \cdot L=\frac{\operatorname{adj} G}{|G|} \cdot L=\left(\begin{array}{cccc}
\operatorname{adj} G_{11} \cdot l_{1} & \operatorname{adj} G_{12} \cdot l_{2} & \cdots & \operatorname{adj} G_{1 n} \cdot l_{n} \\
\operatorname{adj} G_{21} \cdot l_{1} & \operatorname{adj} G_{22} \cdot l_{2} & \cdots & \operatorname{adj} G_{2 n} \cdot l_{n} \\
\vdots & \vdots & \ddots & \vdots \\
\operatorname{adj} G_{n 1} \cdot l_{1} & \operatorname{adj} G_{n 2} \cdot l_{2} & \cdots & \operatorname{adj} G_{n n} \cdot l_{n}
\end{array}\right) /|G|
$$

where $|G(s)|$ is the determinant of $G(s)$, adjG(s) is the adjugate matrix of $G(s)$, that is, the transpose of the cofactor matrix of $\mathrm{G}(\mathrm{s})$, and $\operatorname{adjG}_{\mathrm{ij}}(\mathrm{s})$ is the $\operatorname{adjG}(\mathrm{i}, \mathrm{j})$ element, that is equivalent to the cofactor corresponding to $\mathrm{g}_{\mathrm{ji}}(\mathrm{s})$.

From (3), it can be appreciated that the controller elements of the same column $\mathrm{j}$ of $K(s)$ are related to the same open loop transfer function $l_{j}(s)$. In order to determine the $n \times n$ controller elements $k_{i j}(s)$, it is necessary to specify $n$ open loop transfer functions $l_{j}(s)$. Two aspects must be taken into account when each transfer function $l_{j}(s)$ is defined: the realizability of the controller elements associated to the same column $\mathrm{j}$ and the performance specifications of the corresponding closed loop transfer function $h_{j}(s)$. Since the closed loop response must be stable and without steady state errors due to setpoint or load changes, the open loop transfer function $l_{j}(s)$ must contain an integrator. Then, the general expression (4) is proposed, where the parameter $k_{j}$ becomes a tuning parameter to meet design specifications and the transfer function $\overline{l_{j}}(\mathrm{~s})$ takes into account the realizability requirements.

$$
l_{j}(\mathrm{~s})=\frac{k_{j} \overline{l_{j}}(\mathrm{~s})}{s}
$$

\subsection{Realizability conditions}

The realizability requirement for the controller $\mathrm{K}(\mathrm{s})$ is that all of its elements must be proper, causal and stable. For processes with time delays, non-minimum-phase zeros or 
different relative degrees, direct calculation can lead to elements with prediction, RHP poles or negative relative degrees. According to (3), the expression of a controller element $\mathrm{k}_{\mathrm{ij}}(\mathrm{s})$ is the adjugate element $\operatorname{adj}_{\mathrm{ij}}(\mathrm{s})$ multiplied by the desired open loop transfer function $l_{j}(s)$ and divided by the determinant of the process. As $l_{j}(s)$ is common to all controller elements of the same column, it must be defined taking into account three aspects to be inspected in each column of the inverse of $G(s)$, that is,

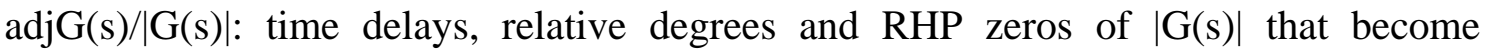
unstable poles.

In order to achieve realizability in the controller elements of the column $\mathrm{j}$, the transfer function $\overline{l_{j}}(\mathrm{~s})$ has the general form given in (5) and it is defined according to the following rules:

- If there are no realizability problems, $\bar{l}_{j}(\mathrm{~s})$ is initially equal to the unity.

- If the non-realizability comes from an element with noncausal time delay, an additional time delay $\theta_{\mathrm{j}}$ is inserted.

- If the non-realizability comes from a RHP zeros $z_{x}$ of $|G(s)|$, which has become unstable pole, this RHP zero is added together with its mirrored pole and the proper multiplicity $\eta_{\mathrm{xj}}$.

- If it comes from a properness problem, a simple stable and fast pole with time constant $\tau$ and the adequate multiplicity $r_{j}$ is inserted.

$$
\overline{l_{j}}(\mathrm{~s})=e^{-\theta_{j} s} \cdot \frac{1}{(\tau s+1)^{r_{j}}} \cdot \prod_{x=1}^{N z}\left(\frac{-s+z_{x}}{s+z_{x}}\right)^{\eta x_{j}}
$$

To achieve realizability in each column $\mathrm{j}$, the minimum dynamics that are necessary to include in $\overline{l_{j}}(\mathrm{~s})$ is given by: 


$$
\begin{array}{cc}
\theta_{j}=\max \left(\max \left(\theta_{G}-\theta_{i j}\right), 0\right) & \forall i \\
r_{j}=\max \left(\max \left(r_{G}-r_{i j}\right)-1,0\right) & \forall i \\
\eta_{x j}=\max \left(\max \left(\eta_{x G}-\eta_{x i j}\right), 0\right) \quad \forall i, \forall x
\end{array}
$$

where $\theta_{i j}$ and $\theta_{G}$ are the time delays of $\operatorname{adjG}_{\mathrm{ij}}(\mathrm{s})$ and $|\mathrm{G}(\mathrm{s})|$, respectively; $r_{i j}$ and $r_{G}$ are their relative degrees; and $\eta_{x i j}$ and $\eta_{x G}$ are the multiplicities of the RHP zero $\mathrm{z}_{\mathrm{x}}$. Equation (7) has been formulated taking into account that the desired open loop process $l_{j}(\mathrm{~s})$ already adds an extra relative degree with the integrator according to (3). Expression (8) must be satisfied for the different RHP zeros of $|G(s)|$.

The conditions (6), (7) and (8) could be formulated with the inverse matrix of $\mathrm{G}(\mathrm{s})$ instead of $\operatorname{adjG(s)}$ and $|\mathrm{G}(\mathrm{s})|$. Nevertheless, the previous notation has been preferred because to carry out the proposed methodology in multivariable time delay systems, it is usually necessary to approximate the expressions of the determinant of the process $|G(s)|$ and its adjugate matrix adjG(s). Although the process dynamics are simple, direct calculations using (3) can give rise to non-rational expressions that are very complicated and difficult to implement. Therefore, before continuing with the design methodology, it is more preferable to approximate such elements by rational transfer functions plus a possible time delay. The proposed approximation method is explained later.

\subsection{Performance specifications}

Next, it is explained how to specify the different parameters of the open loop transfer functions $l_{j}(s)$ when attention is addressed to some of the three simple cases listed in Table 1. Many other cases can be studied; however, the three cases shown in Table 1 arise very often and direct tuning expressions can be obtained from them. Some similar cases 
were studied in (Garrido et al., 2013) where the centralized decoupling control methodology is developed.

Table 1. Three simple cases to define the open loop transfer function.

\begin{tabular}{lll}
\hline Case & $\overline{l_{j}}(\mathrm{~s})$ & $l_{j}(\mathrm{~s})$ \\
\hline 1 & $e^{-\theta_{j} s}$ & $\frac{k_{j}}{s} \cdot e^{-\theta_{j} s}$ \\
2 & $\frac{(-s+z)}{s+z} \cdot e^{-\theta_{j} s}$ & $\frac{k_{j} \cdot(-s+z)}{s \cdot(s+z)} \cdot e^{-\theta_{j} s}$ \\
3 & $\frac{\left(s+z_{j}\right)}{s} \cdot e^{-\theta_{j} s}$ & $\frac{k_{j} \cdot\left(s+z_{j}\right)}{s^{2}} \cdot e^{-\theta_{j} s}$ \\
\hline
\end{tabular}

Firstly, it is preferable to specify simple open loop transfer functions so that their parameters can be easily tuned to meet the desired specifications. It also contributes to achieve simpler controller elements. As many industrial processes can be modelled by first or second order systems, the relative degrees of the process elements of the transfer matrix $G(\mathrm{~s})$ are usually one or two. Therefore, it is very common that the $l_{j}(\mathrm{~s})$ transfer functions need to be specified with relative degree one or two in order to fulfil condition (7), as in the cases of Table 1 . Secondly, some time delay can be necessary in $l_{j}$ (s) to fulfil condition (6). The third case is somewhat special because it is dedicated to processes that show an integrator associated to some output.

\subsubsection{Case 1}

In this case, it is necessary at most a time delay to achieve realizability. The rest of dynamics associated to the column $\mathrm{j}$ of the inverse of $\mathrm{G}(\mathrm{s})$ are stable and minimum phase dynamics, and therefore, they are cancellable. The corresponding expressions for $\overline{l_{j}}(\mathrm{~s})$ and $l_{j}(\mathrm{~s})$ are given in the first row of Table 1 . The imposition of relative stability 
specifications is enough to guarantee the stability of the closed loop transfer function $h_{j}(\mathrm{~s})=l_{j}(\mathrm{~s}) /\left(1+l_{j}(\mathrm{~s})\right)$. It can be found that the phase margin $\varphi_{m}$ and gain margin $A_{m}$ of $l_{j}(\mathrm{~s})$ are given by (9) and (10), respectively, at the corresponding frequencies $\omega_{c p}$ and $\omega_{c g}$ shown in (9) and (10) as well.

$$
\begin{gathered}
\varphi_{m}=90-\frac{180 k_{j} \theta_{j}}{\pi} \quad \omega_{c p}=k_{j} \\
A_{m}=\frac{\pi}{2 k_{j} \theta_{j}} \quad \omega_{c g}=\frac{\pi}{2 \theta_{j}}
\end{gathered}
$$

Both margins are related as follows:

$$
\varphi_{m}=90-\frac{20}{A_{m}}
$$

If a phase margin less than $90^{\circ}$ or a gain margin greater than 1 is specified, the value of $k_{j}$ can be directly calculated by means of (12) or (13), respectively. Increasing $k_{j}$ makes the closed loop response faster. However, it implies smaller values of phase margin and gain margin and consequently, the control system robustness is reduced.

$$
\begin{gathered}
k_{j}=\frac{\pi\left(90-\varphi_{m}\right)}{180 \cdot \theta_{j}} \\
k_{j}=\frac{\pi}{2 A_{m} \theta_{j}}
\end{gathered}
$$

If $\overline{l_{j}}(\mathrm{~s})$ do not have time delay $\left(\theta_{j}=0\right)$ so it is equal to the unity, the open loop function $l_{j}(\mathrm{~s})=k_{j} / \mathrm{s}$ has a phase margin of $90^{\circ}$ and an infinite gain margin independently of the $k_{j}$ parameter. In addition, the closed loop transfer function $h_{j}(\mathrm{~s})$ is a traditional first order system with time constant $T_{j}=1 / k_{j}$, as follows: 


$$
h_{j}(s)=\frac{k_{j} / s}{k_{j} / s+1}=\frac{1}{T_{j} s+1}
$$

Therefore, the desired closed loop time constant $T_{j}$ is proposed as specification to determine $k_{j}$ instead of relative stability margins. This situation is equivalent to one of the most common cases in the methodologies of IMC control (Rivera, Morari, \& Skogestad, 1986) or affine parameterization (Goodwin, Graebe, \& Salgado, 2001).

\subsubsection{Case 2}

In this case, in addition to a possible time delay, it is necessary to specify in $\overline{l_{j}}(\mathrm{~s})$ a nonminimum phase zero $\mathrm{z}$ of the determinant of the process $|\mathrm{G}(\mathrm{s})|$. To achieve internal stability, this multivariable RHP zero should be included into the open loop processes. When this zero does not appear in the adjG(s) elements and its multiplicity in $|G(s)|$ is the unity, it is included together with its mirrored polo in order to fulfill condition (8). The expressions of $\overline{l_{j}}(\mathrm{~s})$ and $l_{j}(\mathrm{~s})$ are given in the second row of Table 1 .

In these conditions, it can be demonstrated that gain margin of $l_{j}(s)$ is given by (15) at the frequency $\omega_{c g}$ that fulfills condition (16). The frequency $\omega_{c g}$ can be obtained from (16) and then, the gain $\mathrm{k}_{\mathrm{j}}$ is calculated from (15) in order to achieve the desired gain margin.

$$
\begin{aligned}
A_{m} & =\frac{\omega_{c g}}{k_{j}} \\
\tan \left(\omega_{c g} \theta_{j}\right) & =\frac{1-\omega_{c g}^{2} / z^{2}}{2 \omega_{c g} / z}
\end{aligned}
$$


If there are no time delays in $l_{j}(\mathrm{~s})\left(\theta_{j}=0\right)$, the gain margin specification can be replaced by time response specifications because the corresponding closed loop transfer function $h_{j}(\mathrm{~s})$ is given by a second order system as follows:

$$
h_{j}(s)=\frac{\frac{k_{j}(-s+z)}{s(s+z)}}{1+\frac{k_{j}(-s+z)}{s(s+z)}}=\frac{k_{j}(-s+z)}{s^{2}+\left(z-k_{j}\right) s+k_{j} z}
$$

The poles of $h_{j}(\mathrm{~s})$ in (17) are characterized by the undamped natural frequency $\omega_{n}$ and the damping factor $\xi$ given by (18). Therefore, it is possible to fix the value of $\xi$ with $k_{j}<z$.

$$
\omega_{n}=\sqrt{k_{j} z} \quad \xi=\frac{z-k_{j}}{2 \sqrt{k_{j} z}}
$$

\subsubsection{Case 3}

This special case appears when all elements of the column $j$ of the inverse of $G(s)$ consist of a zero at $s=0$, which is usually associated to an output with an integrator. An extra integrator should be specified in $\overline{l_{j}}(\mathrm{~s})$ in order to maintain the integral action in the $\mathrm{k}_{\mathrm{ij}}(\mathrm{s})$ elements of the column $\mathrm{j}$ of $\mathrm{K}(\mathrm{s})$. Otherwise, the integrator would be cancelled in the corresponding controller elements and consequently, zero error in steady state would not be guaranteed in that loop. Because of this additional integrator, the relative degree of $l_{j}(\mathrm{~s})$ increases one unit. If no additional degrees are needed, an extra zero $z_{j}$ can be included to keep the proper relative degree in $l_{j}(\mathrm{~s})$ and fulfill condition (7). An additional time delay can be needed to fulfill condition (6). Then, the corresponding expressions for $\overline{l_{j}}(\mathrm{~s})$ and $l_{j}(\mathrm{~s})$ are given by the third row of Table 1 . In these conditions, the gain margin of $l_{j}(\mathrm{~s})$ is given by (19) at the frequency $\omega_{c g}$ that fulfills condition (20). 
Authors propose to fix the value of the zero $z_{j}$, subsequently, to calculate the frequency $\omega_{c g}$ from (20) and finally, to obtain the parameter $k_{j}$ from (19).

$$
\begin{gathered}
A_{m}=\frac{\omega_{c g}^{2}}{k_{j} \sqrt{\omega_{c g}^{2}+z_{j}^{2}}} \\
\arctan \left(\frac{\omega_{c g}}{z_{j}}\right)-\omega_{c g} \theta_{j}=0
\end{gathered}
$$

If $\overline{l_{j}}(\mathrm{~s})$ do not contain time delays, the gain margin specification can be replaced again by time response specifications since the closed loop transfer function is given by a second order transfer function with a zero at $s=-z_{j}$, as shown in (21). Its poles are characterized by the natural frequency $\omega_{n}$ and the damping factor $\xi$ given by (22). Therefore, fixing the value of the zero $z_{j}$, it is possible to modify the values of $\omega_{n}$ and $\xi$ through the parameter $k_{j}$. As a particular case, adjusting $k_{j}=4 \cdot z_{j}$, a critical damping response $(\xi=1)$ is obtained with $\omega_{n}=2 \cdot z_{j}$.

$$
\begin{gathered}
h_{j}(s)=\frac{k_{j}\left(s+z_{j}\right) / s^{2}}{1+k_{j}\left(s+z_{j}\right) / s^{2}}=\frac{k_{j}\left(s+z_{j}\right)}{s^{2}+k_{j} s+k_{j} z_{j}} \\
\omega_{n}=\sqrt{k_{j} z_{j}} \quad \xi=\sqrt{\frac{k_{j}}{4 z_{j}}}
\end{gathered}
$$

In the three explained cases, the parameter $k_{j}$, which is common to the $\mathrm{k}_{\mathrm{ij}}(\mathrm{s})$ elements of the column $\mathrm{j}$ of $\mathrm{K}(\mathrm{s})$, acts as a degree of freedom to modify the corresponding closed loop $\mathrm{j}$ performance and to achieve new specifications almost independently of the other loops. 


\subsection{Formulation for $2 \times 2$ processes}

In this section, the expressions of the proposed method are presented for the particular case of $2 \times 2$ systems, where the decoupling control scheme is depicted in Figure 2 . Assume the process transfer matrix is given by (23), where each element $g_{i j}(\mathrm{~s})$ is split into two parts: the free time delay transfer function $g_{o i j}(\mathrm{~s})$ and the part with the corresponding time delay $\theta_{i j}$. With this notation, the general expression of the controller matrix K(s) is given by (24), where the Laplace variable s is omitted.

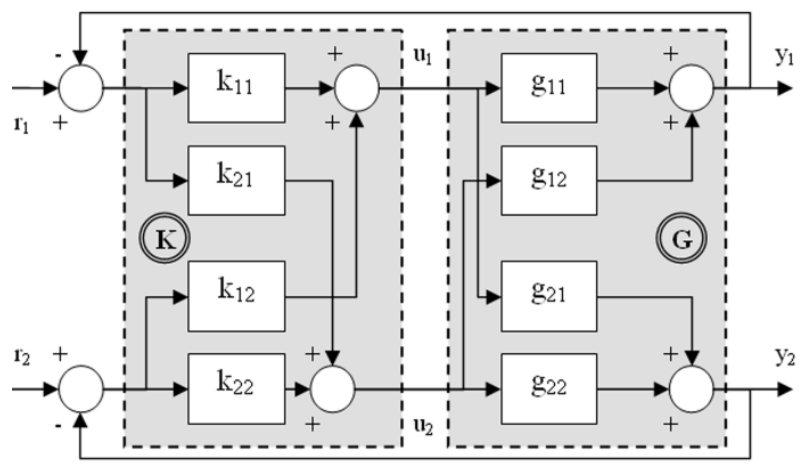

Figure 2. Centralized decoupling control scheme for $2 \times 2$ processes

$$
\begin{gathered}
G(\mathrm{~s})=\left(\begin{array}{ll}
g_{o 11}(\mathrm{~s}) e^{-\theta_{11} s} & g_{o 12}(\mathrm{~s}) e^{-\theta_{12} s} \\
g_{o 21}(\mathrm{~s}) e^{-\theta_{21} s} & g_{o 22}(\mathrm{~s}) e^{-\theta_{22} s}
\end{array}\right) \\
K=\frac{\left(\begin{array}{cc}
g_{o 22} e^{-\theta_{22} s} l_{1} & -g_{o 12} e^{-\theta_{12} s} l_{2} \\
-g_{o 21} e^{-\theta_{21} s} l_{1} & g_{o 11} e^{-\theta_{11} s} l_{2}
\end{array}\right)}{g_{o 11} g_{o 22} e^{-\left(\theta_{11}+\theta_{22}\right) s}-g_{o 12} g_{o 21} e^{-\left(\theta_{12}+\theta_{21}\right) s}}
\end{gathered}
$$

Before continuing with the design in systems with time delays, it is necessary to approximate the determinant $|\mathrm{G}(\mathrm{s})|$ by a rational transfer function $\chi_{o}(\mathrm{~s})$ plus a time delay $\theta_{G}$, which is given by (25) assuming nonzero process elements.

$$
\theta_{G}=\min \left(\theta_{11}+\theta_{22}, \theta_{12}+\theta_{21}\right)
$$


After the approximation, the necessary dynamics in the $\bar{l}_{j}(\mathrm{~s})$ elements to achieve realizability can be determined. For instance, the minimum time delays $\theta_{1}$ and $\theta_{2}$ which have to be specified in $\overline{l_{1}}(\mathrm{~s})$ and $\overline{l_{2}}(\mathrm{~s})$ are given by (26). The general expressions of the controller elements are shown in (27). The four elements have integral action, and at least, two of them must delay their control action in case of processes with time delays.

$$
\begin{gathered}
\theta_{1}=\max \left(\theta_{G}-\min \left(\theta_{22}, \theta_{21}\right), 0\right) \\
\theta_{2}=\max \left(\theta_{G}-\min \left(\theta_{12}, \theta_{11}\right), 0\right) \\
K(\mathrm{~s})=\left(\begin{array}{cc}
k_{1} \frac{g_{o 22}(\mathrm{~s}) \overline{l_{1}}(\mathrm{~s})}{s \cdot \chi_{o}(\mathrm{~s})} e^{-\left(\theta_{22}-\theta_{G}\right) s} & -k_{2} \frac{g_{o 12}(\mathrm{~s}) \overline{l_{2}}(\mathrm{~s})}{s \cdot \chi_{o}(\mathrm{~s})} e^{-\left(\theta_{12}-\theta_{G}\right) s} \\
-k_{1} \frac{g_{o 21}(\mathrm{~s}) \overline{l_{1}}(\mathrm{~s})}{s \cdot \chi_{o}(\mathrm{~s})} e^{-\left(\theta_{21}-\theta_{G}\right) s} & k_{2} \frac{g_{o 11}(\mathrm{~s}) \overline{l_{2}}(\mathrm{~s})}{s \cdot \chi_{o}(\mathrm{~s})} e^{-\left(\theta_{11}-\theta_{G}\right) s}
\end{array}\right)
\end{gathered}
$$

\subsection{Multivariable PID control}

The resultant controller elements in the proposed methodology can be quite complex and difficult to implement not only for higher dimensional systems but also for $2 \times 2$ processes, as it can be appreciated from (27). In this work, a PID reduction is carried out for each controller element in $\mathrm{K}(\mathrm{s})$ and, as a result, a centralized PID control is obtained. This PID reduction is explained later in Section 3.

As result, the proposed methodology for $n \times n$ processes can be summarized in the following steps:

(1) Obtain time delays, relative degrees and RHP zeros and its multiplicity of $|G(s)|$ and $\operatorname{adjG(s)~elements~from~the~process~transfer~matrix~} G(s)$. In case of time delay systems, it is generally necessary to approximate $|G(s)|$ and adjG(s) 
elements into rational transfer functions before obtaining this information. The approximation method in section 3.1 is proposed.

(2) According to the realizability conditions (6), (7) and (8), determine the necessary dynamics (time delay, extra poles and RHP zeros, respectively) to be included in the corresponding desired open loop process $l_{j}(\mathrm{~s})$ in order to achieve realizability into the centralized controller. The proposed form of open loop transfer function is given by (5). It has an integrator to obtain no steady state error due to setpoint or load step changes.

(3) Adjust the gain parameter $k_{j}$ in the open loop processes $l_{j}(\mathrm{~s})$ to achieve the performance specifications. If the transfer function $l_{j}(\mathrm{~s})$ matches some of the three studied cases in Table 1, perform the tuning using the corresponding expressions proposed in section 2.2.

(4) Calculate the centralized decoupling control $\mathrm{K}(\mathrm{s})$ with integral action that minimizes the interaction by means of (3).

(5) Approximate the controller elements to PID structure, obtaining $\mathrm{K}^{\mathrm{PID}}$ (s). The gains $k_{j}$ can be used as degrees of freedom in each loop $\mathrm{j}$ to modify the performance specifications. In order to test other values of $\mathrm{k}_{\mathrm{j}}$ it is not necessary to repeat the whole procedure. It is enough to transmit the change rate to gains of the $\mathrm{K}(\mathrm{s})$ elements of the corresponding column $\mathrm{j}$.

(6) Because of the two approximations carried out so far in the proposed method, it is suggested to assure the stability and robustness of the control systems as detailed in the following section 2.5.

(7) From a practical point of view, implement the centralized controller using the new scheme proposed in section 3.3 for multivariable PID controllers. It avoids 
windup problem and allows bumpless transfer between automatic and manual operation modes.

\subsection{Stability and robustness}

The first condition for stability that the final controller K(s) must fulfill is that all of its elements must not have RHP poles. Once the stability of the controller is assured, internal stability of the closed loop system can be verified if and only if all elements in matrix (28) have all their poles in the left-half plane with no RHP pole-zero cancellation in $\mathrm{G}(\mathrm{s}) \cdot \mathrm{K}(\mathrm{s})$ (Skogestad \& Postlethwaite, 2005). In this case, S(s) is the sensitivity transfer function matrix written as $[\mathrm{I}+\mathrm{G}(\mathrm{s}) \cdot \mathrm{K}(\mathrm{s})]^{-1}$.

$$
\left[\begin{array}{cc}
(I+K(s) \cdot G(s))^{-1} & -K(s) \cdot S(s) \\
S(s) G(s) & S(s)
\end{array}\right]
$$

The K(s) elements obtained with the proposed method are more complex than those obtained using centralized inverted decoupling. In processes with time delays, approximations are needed, which is an important disadvantage over centralized inverted decoupling. Therefore, centralized inverted decoupling is preferable when applicable. However, it cannot be applied to processes with RHP zeros in the determinant. Fortunately, the proposed methodology can be applied in these cases because these RHP zeros can be included in the open loop process $\mathrm{L}(\mathrm{s})$ according to the case 2 of Table 1.

To evaluate the robustness of the controller, a $\mu$-analysis can be performed for different types of uncertainties. For instance, multiplicative input uncertainty is illustrated in Figure 3, where $\Delta_{\mathrm{I}}(\mathrm{s})$ is the disturbance and $\mathrm{W}_{\mathrm{I}}(\mathrm{s})$ and $\mathrm{W}_{\mathrm{P}}(\mathrm{s})$ are the diagonal weights for uncertainty and performance, respectively. 


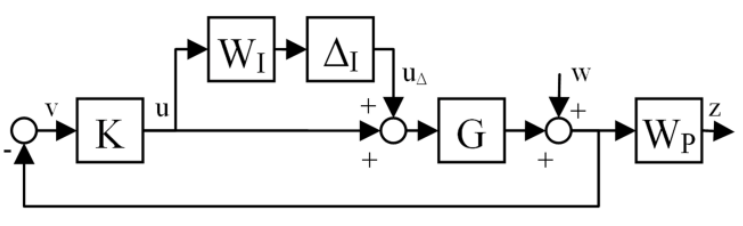

Figure 3. System with multiplicative input uncertainty and performance measured at the output.

To achieve robust stability, the necessary and sufficient condition (Skogestad \& Postlethwaite, 2005) is

$$
\mu_{\mathrm{RS}}=\mu\left[-\mathrm{W}_{\mathrm{I}}(\mathrm{s}) \mathrm{T}_{\mathrm{I}}(\mathrm{s})\right]<1 \quad \forall \omega
$$

where $\mu$ is the structured singular value (SSV) and $T_{I}(s)$ (equal to $K(s) G(s)\left(I+K(s) G(s){ }^{-}\right.$ ${ }^{1}$ ) is the input complementary sensitivity function. To evaluate whether the closed loop system will respect the desired performance even in the presence of diagonal multiplicative input uncertainty, the necessary and sufficient condition (Skogestad \& Postlethwaite, 2005) is

$$
\mu_{\mathrm{RP}}=\mu\left[\begin{array}{cc}
-\mathrm{W}_{\mathrm{I}}(\mathrm{s}) \mathrm{T}_{\mathrm{I}}(\mathrm{s}) & -\mathrm{W}_{\mathrm{I}}(\mathrm{s}) \mathrm{K}(\mathrm{s}) \mathrm{S}(\mathrm{s}) \\
\mathrm{W}_{\mathrm{P}}(\mathrm{s}) \mathrm{S}(\mathrm{s}) \mathrm{G}(\mathrm{s}) & \mathrm{W}_{\mathrm{P}}(\mathrm{s}) \mathrm{S}(\mathrm{s})
\end{array}\right]<1 \quad \forall \omega
$$

\section{Aproximation methods and anti-windup scheme}

In this section, some design and practical considerations are discussed in a similar way to that described in (Garrido et al., 2012).

\subsection{Approximation of $|G(s)|$ and adjG(s)}

To carry out the proposed methodology in multivariable time delay systems, it is usually necessary to approximate the expressions of the determinant of the process $|G(s)|$ and its adjugate matrix adjG(s). Although the process dynamics are simple, direct calculations can give rise to non-rational expressions that are very complicated and 
difficult to implement (as shown in (31)):

$$
F(s)=\frac{\left(-0.0089 \cdot(5.6 s+1) \cdot e^{-2 s}+0.0051 \cdot(10 s+1)\right)}{(5.6 s+1) \cdot(8 s+1) \cdot(10 s+1)} e^{-3 s}
$$

Therefore, before continuing with the design methodology, it is more preferable to approximate such elements by rational transfer functions plus a possible time delay. Because it is easy to obtain the frequency response of $|G(j w)|$ and adjG(jw) from the process frequency response $G(j w)$, a parametric approximation in the frequency domain is proposed. Several techniques can be used for this purpose. Some are based on least squares estimators (Pintelon, Guillaume, Rolain, Schoukens, \& Van hamme, 1994); others, such as the prediction error method (PEM), are based on an iterative estimation method that minimizes the prediction errors to obtain maximum likelihood estimates.

In this work, a simple linear least square approximation is proposed in the frequency range of interest as an example. The lowest frequency is chosen two decades below the smallest absolute value of the real part of the different poles and zeros of G(s); the highest frequency is chosen two decades above the greatest one. The procedure is shown in the following steps.

\subsubsection{Previous information}

To facilitate the approximation, the following previous information about the function is obtained from the Bode plots of its frequency response and from $G(s)$ :

- External time delay. A general expression for the non-zero element of $\operatorname{adj}_{\mathrm{ij}}(\mathrm{s})$ or $|\mathrm{G}(\mathrm{s})|$ is (Wang et al., 2002):

$$
\chi(s)=\sum_{k=1}^{M} \chi_{k}(s) \cdot e^{-\theta_{k} s} \quad \theta_{k} \geq 0
$$


where $\chi_{\mathrm{k}}(\mathrm{s})$ is a non-zero scalar rational transfer function that is obtained as a product of some process transfer functions $\mathrm{g}_{\mathrm{ij}}(\mathrm{s})$; the corresponding time delay $\theta_{\mathrm{k}}$ is the sum of the time delays of these transfer functions $g_{\mathrm{ij}}(\mathrm{s})$. Therefore, the time delay for $\chi(\mathrm{s})$ is defined as follows

$$
\tau(\chi(s))=\min _{k=1, \ldots, M}\left(\theta_{k}\right)
$$

From the time delay matrix of $G(s)$, the corresponding time delays of $|G(s)|$ and adjG(s) can then be derived.

- Relative degree. This value can be calculated from the high frequency roll-off of the module Bode plot. If the relative degree is $r d$, the rate of roll-off will be 20. $r d \mathrm{~dB} /$ decade. Even though this rate is not exactly constant because of the non-rational nature of the function to be approximated, a general trend can be estimated (in $\mathrm{dB} /$ decade) from the frequency response of the $n$ data points in the last decade. The estimation is given by (34) using least squares, where $\mathrm{y}_{\mathrm{i}}$ is the module (in $\mathrm{dB}$ ) of the response for the frequency $\mathrm{w}_{\mathrm{i}}$ (in decades). Then, the relative degree $r d$ is obtained from (35) and rounded to the closest integer.

$$
\begin{gathered}
\text { roll - off }=\frac{n \cdot \sum_{i=1}^{n} w_{i} \cdot y_{i}-\sum_{i=1}^{n} w_{i} \cdot \sum_{i=1}^{n} y_{i}}{n \cdot \sum_{i=1}^{n} w_{i} \cdot w_{i}-\left(\sum_{i=1}^{n} w_{i} \cdot w_{i}\right)^{2}} \\
\mathrm{rd}=\frac{\text { rool }- \text { off }}{-20}
\end{gathered}
$$

- Original poles. The determinant $|\mathrm{G}(\mathrm{s})|$ and the elements of adjG(s) are the result of the sums and products of different elements of $\mathrm{G}(\mathrm{s})$. Thus, assuming that 
there are no pole-zero cancellations after these operations, the poles of $|G(s)|$ and $\operatorname{adjG}_{\mathrm{ij}}(\mathrm{s})$ can be calculated. The integrators (poles in $\mathrm{s}=0$ ) will be removed from the frequency response before the approximation.

- Number of RHP zeros. After removing the previous external time delay and integrators from the frequency response, the number of RHP zeros can be estimated from the phase shift in the phase Bode plot if the phase at high frequencies oscillates around a constant value. Assuming that the relative degree $r d$, the low frequency phase $\varphi_{0}$ and high frequency phase $\varphi_{\text {end }}$ are known, the number of RHP zeros $\mathrm{N}_{\mathrm{z}}$ in this case can be obtained from (36):

$$
N_{z}=\frac{\varphi_{\text {end }}-\varphi_{o}+90^{\circ} \cdot r d}{-180^{\circ}}
$$

As an example, Figure 4 shows the Bode plots of the irrational transfer function in (31) after removing the external time delay of three units and the stationary gain equal to -0.0038 . The corresponding approximation is also shown. As previously commented, the rate of roll-off at high frequencies is not entirely constant; however, a trend of approximately $-40 \mathrm{~dB} /$ decade can be observed, and a relative degree equal to 2 is obtained using (35). Similarly, the phase at high frequencies oscillates; in this case, it oscillates around a constant phase of $-360^{\circ}$. Therefore, expression (36) indicates that the approximation model should have a RHP zero.

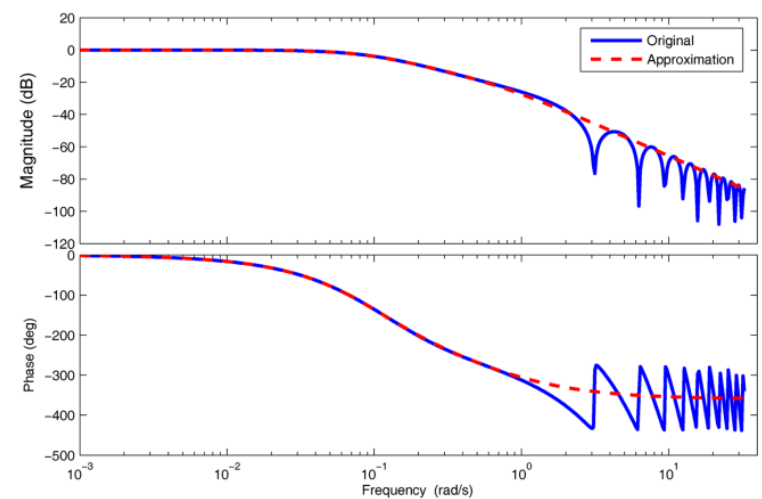


Figure 4. Bode plots of expression (31) after removing the time delay and stationary gain, and its corresponding approximation.

Nevertheless, the high frequency phase at several instances does not tend to a constant value and the previous estimation was not valid because of the irrationality of some functions that were to be approximated. Therefore, $\mathrm{N}_{\mathrm{z}}$ is assumed to be zero and the existence of RHP zeros will depend on the approximation.

\subsubsection{Simplifying the approximation and order estimations}

Before carrying out the least square approximation, the time delay and possible integrators are removed from the frequency response data in order to simplify the approximation. Additionally, this new response is divided by its stationary gain; this results in the new frequency response $\mathrm{M}(\mathrm{jw})$ to be approximated.

In the simplest parametric model for the approximation of $\mathrm{M}(\mathrm{jw})$, the denominator order is equal to the relative degree plus the number of RHP zeros; its numerator order is the number of RHP zeros. In the most complex parametric model, the denominator order is the number of original poles, and the numerator order is equal to denominator order minus the relative degree. Note that the relative degree must be updated if any integrator has been removed.

The approximation method is applied to the simplest model. If the method does not produce a good fit, the orders are progressively increased until a good fit is obtained or the most complex model is reached. In the latter case or when unstable models are obtained, the tolerance for a good fit is relaxed, and the process is restarted. After achieving a good estimated model $\mathrm{N}(\mathrm{s}) / \mathrm{D}(\mathrm{s})$, the removed time delay, stationary gain and possible integrators are added back in. 


\subsubsection{Approximation method}

Assuming that the frequency response $\mathrm{M}(\mathrm{jw})$ is intended to be approximated by a model $\mathrm{N}(\mathrm{s}) / \mathrm{D}(\mathrm{s})$ with even orders $\mathrm{p}$ and $\mathrm{q}$ in the numerator and denominator respectively, the corresponding rational transfer function to be estimated with unitary stationary gain, is given by

$$
\frac{\mathrm{N}(\mathrm{s})}{\mathrm{D}(\mathrm{s})}=\frac{\alpha_{p} s^{p}+\ldots+\alpha_{2} s^{2}+\alpha_{1} s+1}{\beta_{q} s^{2 q}+\ldots+\beta_{2} s^{2}+\beta_{1} s+1}
$$

The proposed weighted linear least square method tries to minimize the following cost function $\mathrm{J}$ in (38), where $\mathrm{W}(\mathrm{jw}, \beta)$ given in (39) is a weighting function to minimize the relative error criterion (Pintelon, 94):

$$
\begin{gathered}
\min J=\sum_{\mathrm{k}=1}^{\mathrm{n}} \mathrm{W}\left(\mathrm{jw}_{\mathrm{k}}, \beta\right) \cdot\left|\mathrm{M}\left(\mathrm{jw}_{\mathrm{k}}\right) \cdot \mathrm{D}\left(\mathrm{jw}_{\mathrm{k}}, \beta\right)-\mathrm{N}\left(\mathrm{jw}_{\mathrm{k}}, \alpha\right)\right|^{2} \\
\mathrm{~W}\left(\mathrm{jw}_{\mathrm{k}}, \beta^{\mathrm{i}}\right)=\frac{1}{\left|\mathrm{M}\left(\mathrm{jw}_{\mathrm{k}}\right) \cdot \mathrm{D}\left(\mathrm{jw}_{\mathrm{k}}, \beta^{\mathrm{i}-1}\right)\right|}
\end{gathered}
$$

If the previous problem is decomposed into real and imaginary parts, it can be

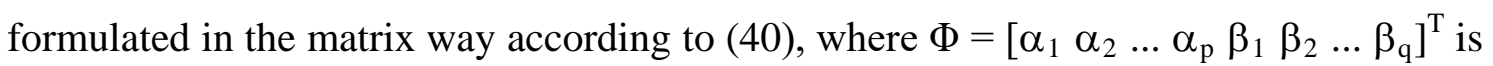
the parameter vector to be estimated, $\mathrm{W}$ is a weighting diagonal matrix, $\mathrm{b}$ is a vector that depends on the frequency response data, and $\mathrm{A}$ is a matrix depending on the frequency response data and the order of the model (37) as shown below. Once the problem is stated according to (40), the solution will be given by (41).

$$
\begin{aligned}
\min \mathrm{J} & =(\mathrm{b}-\mathrm{A} \cdot \Phi)^{\mathrm{T}} \cdot \mathrm{W} \cdot(\mathrm{b}-\mathrm{A} \cdot \Phi) \\
\Phi & =\left(\mathrm{A}^{\mathrm{T}} \cdot \mathrm{W} \cdot \mathrm{A}\right)^{-1} \mathrm{~A}^{\mathrm{T}} \cdot \mathrm{W} \cdot \mathrm{b}
\end{aligned}
$$


In the ideal case of perfect matching, expression (42) should be fulfilled for each frequency $\mathrm{w}_{\mathrm{k}}$. It can be expanded as shown in (43).

$$
\begin{gathered}
M\left(j w_{k}\right) \cdot D\left(j w_{k}, \beta\right)-N\left(j w_{k}, \alpha\right)=0 \\
M\left(j w_{k}\right) \cdot\left(1 \beta_{1} j w_{k} \beta_{2}\left(j w_{k}\right)^{2} \ldots \beta_{q-1}\left(j w_{k}\right)^{q-1} \beta_{q}\left(j w_{k}\right)^{q}\right) \\
-\left(1 \alpha_{1} j w_{k} \alpha_{2}\left(j w_{k}\right)^{2} \ldots \alpha_{p-1}\left(j w_{k}\right)^{p-1} \alpha_{p}\left(j w_{k}\right)^{p}\right)=0
\end{gathered}
$$

If the function $\psi^{\mathrm{n}}$ is defined according to (44), equation (43) can be expressed in a more compact way shown in (45).

$$
\begin{aligned}
& \psi_{\mathrm{k}}^{\mathrm{n}}\left\{\begin{array}{l}
\operatorname{Re}\left(\mathrm{jw}_{\mathrm{k}}\right)^{\mathrm{n}} \text { if } n \text { is even } \\
\operatorname{Im}\left(\mathrm{jw}_{\mathrm{k}}\right)^{\mathrm{n}} \text { if } n \text { is odd }
\end{array}\right. \\
& \left(\mathrm{M}\left(\mathrm{jw} w_{\mathrm{k}}\right) \cdot\left(\mathrm{j} \psi_{\mathrm{k}}^{1} \psi_{\mathrm{k}}^{2} \ldots \mathrm{j} \psi_{\mathrm{k}}^{\mathrm{q}-1} \psi_{\mathrm{k}}^{\mathrm{q}}\right) \mid-\mathrm{j} \psi_{\mathrm{k}}^{1}-\psi_{\mathrm{k}}^{2} \ldots-\mathrm{j} \psi_{\mathrm{k}}^{\mathrm{p}-1}-\psi_{\mathrm{k}}^{\mathrm{p}}\right) \cdot\left(\begin{array}{l}
\beta_{1} \\
\beta_{2} \\
\vdots \\
\beta_{\mathrm{q}-1} \\
\beta_{\mathrm{q}} \\
\alpha_{1} \\
\alpha_{2} \\
\vdots \\
\alpha_{p-1} \\
\alpha_{p}
\end{array}\right)=1-\mathrm{M}\left(\mathrm{jw} w_{\mathrm{k}}\right)
\end{aligned}
$$

Decomposing (45) into real and imaginary parts and $M\left(j w_{k}\right)$ into $x_{k}+j y_{k}$, the following matrix equation can be obtained:

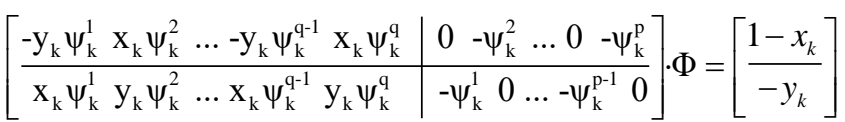

$$
\begin{aligned}
& A_{k} \cdot \Phi=b_{k}
\end{aligned}
$$

Finally, after finding the matrix $A_{k}$ and $b_{k}$ for each frequency $w_{k}$, the entire matrix A and b of expressions (40) or (41) can be formed, and the least square 
approximation can be performed to obtain the estimated parameter vector $\Phi$ that defines the model $\mathrm{N}(\mathrm{s}) / \mathrm{D}(\mathrm{s})$.

The measurement used to determine whether the estimated model is "good" is the mean squared error (MSE), which can be calculated according to (47), where $n$ is the number of elements in the frequency response. If the MSE is under the pre-specified tolerance, the approximation model is acceptable. Initially, the tolerance is $10^{-5}$.

$$
\operatorname{MSE}=b^{T} \cdot\left[W-W \cdot A \cdot\left(A^{T} \cdot W \cdot A\right)^{-1} \cdot A^{T} \cdot W\right] \cdot b /(n-q-p)
$$

For instance, the minimum orders of denominator and numerator model in the case of expression (31) according to its information obtained previously should be three and one, respectively; in addition, the two values coincide with the maximum model orders. After removing the time delay and stationary gain and applying the proposed method, the approximated model with Bode plots depicted in Figure 4 is obtained. It shows a good fit in comparison with the corresponding original frequency response with a mean squared error of $8.39 \cdot 10^{-5}$. Then, the final model in (48) is obtained after adding back the time delay and the original stationary gain.

$$
F_{a p}(\mathrm{~s})=\frac{(-0.0038)(-7.54 \mathrm{~s}+1)}{\left(143.4 \mathrm{~s}^{3}+131.1 \mathrm{~s}^{2}+20.93 \mathrm{~s}+1\right)} \mathrm{e}^{-3 \mathrm{~s}}
$$

\subsection{Controller reduction to multivariable PID}

After approximation, $|\mathrm{G}(\mathrm{s})|$ and all of elements of adjG(s) are expressed as a rational

transfer function plus time delay. Therefore, the necessary dynamics in $\overline{l_{j}}(\mathrm{~s})$ can be determined analyzing the realizability conditions (6), (7) and (8). Then, after finishing the design, the elements of $\mathrm{K}(\mathrm{s})$ are obtained. 
As previously mentioned in subsection 2.4, all of the controller elements of K(s) must be approximated by PID controllers to achieve a centralized PID controller. The PID structure used in this work is the parallel form of (49), where $K_{P}$ is the proportional constant, $K_{I}$ the integral constant, $K_{D}$ the derivative constant and $T_{F}$ is the derivative filter constant. Although the parameters have little physical interpretation in this form, it is the most flexible structure that allows independency between the different control actions.

$$
\mathrm{k}^{\mathrm{PID}}(\mathrm{s})=K_{P}+\frac{K_{I}}{s}+\frac{K_{D} s}{T_{F} s+1}
$$

The PID controller can be obtained using the approximation method described in subsection 3.1. However, the authors propose to remove the integrator of the controller element $\mathrm{k}_{\mathrm{ij}}(\mathrm{s})$ and apply the model reduction to the inverse of this result $\mathrm{t}(\mathrm{s})$ instead of reducing the controller element. The new stationary gain $\mathrm{k}_{0}$, as shown in (50), would be identified with the integral constant $\mathrm{K}_{\mathrm{I}}$.

$$
\mathrm{k}_{0}=\lim _{\mathrm{s} \rightarrow 0}[\mathrm{k}(\mathrm{s}) \cdot \mathrm{s}]=\lim _{\mathrm{s} \rightarrow 0}[\mathrm{t}(\mathrm{s})]
$$

Without the integrator and after dividing by $\mathrm{k}_{0}$, the frequency response of the inverse of $t(s)$ should be approximated according to (51):

$$
\frac{\mathrm{k}_{0}}{\mathrm{t}(\mathrm{s})} \approx \frac{\mathrm{b}_{1} \mathrm{~s}+1}{\mathrm{a}_{2} \mathrm{~s}^{2}+\mathrm{a}_{1} \mathrm{~s}+1}=\frac{\mathrm{T}_{\mathrm{F}} \mathrm{s}+1}{\left(\frac{\mathrm{K}_{\mathrm{P}} \mathrm{T}_{\mathrm{F}}+\mathrm{K}_{\mathrm{D}}}{\mathrm{K}_{\mathrm{I}}}\right) \mathrm{s}^{2}+\left(\frac{\mathrm{K}_{\mathrm{P}}}{\mathrm{K}_{\mathrm{I}}}+\mathrm{T}_{\mathrm{F}}\right) \mathrm{s}+1}
$$

In this way, the PID parameters after approximation can be identified as follows 


$$
\begin{aligned}
& \mathrm{K}_{\mathrm{P}}=\left(\mathrm{a}_{1}-\mathrm{b}_{1}\right) \cdot \mathrm{k}_{0} \\
& \mathrm{~K}_{\mathrm{I}}=\mathrm{k}_{0} \\
& \mathrm{~K}_{\mathrm{D}}=\mathrm{a}_{2} \cdot \mathrm{k}_{0}-\mathrm{b}_{1} \cdot\left(\mathrm{a}_{1}-\mathrm{b}_{1}\right) \cdot \mathrm{k}_{0} \\
& \mathrm{~T}_{\mathrm{F}}=\mathrm{b}_{1}
\end{aligned}
$$

For PI approximation, $\mathrm{K}_{\mathrm{D}}$ and $\mathrm{T}_{\mathrm{F}}$ are removed; therefore, the coefficients $\mathrm{b}_{1}$ and $\mathrm{a}_{2}$ would be zero. Both PI and PID approximations are obtained, the one with the best fit is chosen.

There are multivariable processes that are too complex to be controlled by a multivariable PID controller; the controllers would at least have difficulties fulfilling very restrictive specifications in decoupling and loop performances. In these cases, the specifications should be relaxed to make the response more sluggish; alternatively, higher order or more advanced controllers should be used (Wang, 2003).

\subsection{Anti-windup scheme}

From an implementation point of view, it is important to consider how to solve practical problems such as wind-up, which can cause the controller to perform poorly in the presence of control signal constraints. For monovariable PID controllers, the simple anti-windup scheme in Figure 5 can be used. This scheme is based on back-calculations (Áström \& Hägglund, 2006). It uses an input constraint model inside the controller. When the process input is saturated resulting in a different value than the PID output, the controller works in tracking mode following the saturated signal. This configuration can be found as a PID function block in most commercial distributed control systems (DCS). 


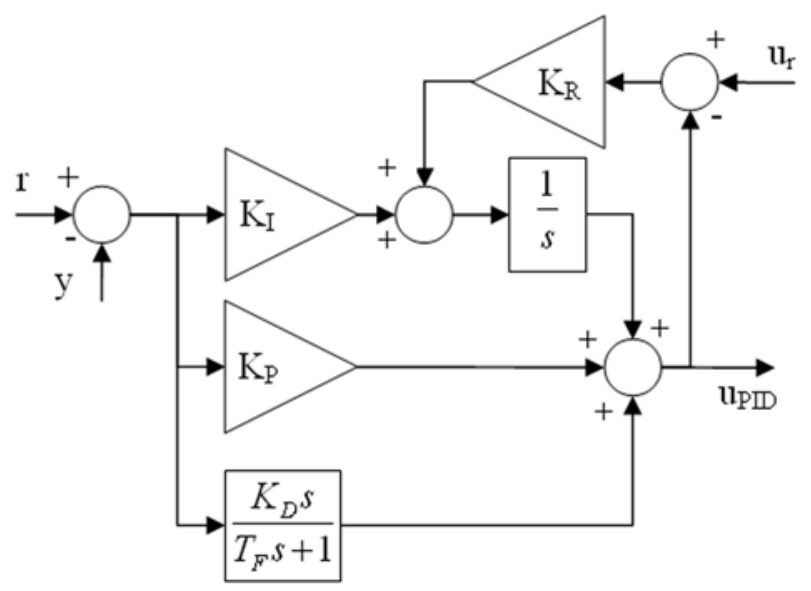

Figure 5. Block diagram of a PID controller with anti-windup using a tracking signal.

Nevertheless, it is more difficult to find wind-up solutions for the multivariable case. In a multivariable PID control system $\mathrm{K}^{\mathrm{PID}}(\mathrm{s})$ in which all of its elements have PID structures, each manipulated variable is composed by the sum of several PID actions. In this case, the wind-up problem becomes more difficult to handle because the contribution of each PID controller to the corresponding process input is difficult to coordinate when saturation occurs.

For a multivariable PID control system with two inputs and two outputs, the anti-windup scheme depicted in Figure 6 is proposed. Although no commercial solution uses this scheme, it can be implemented using function blocks of a distributed control system. Even though it is presented for TITO control systems, they can be extended to higher number of inputs and outputs. 


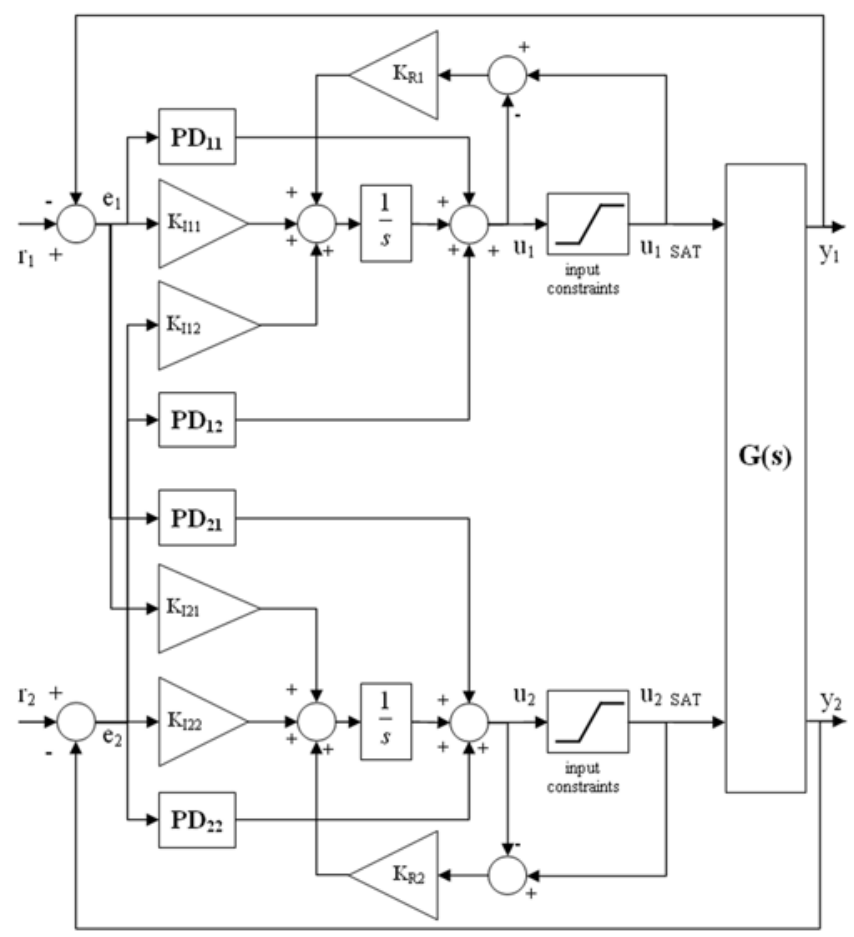

Figure 6. Anti-windup scheme for a multivariable PID controller in a TITO system.

The key idea is to use only one integrator for each manipulated variable so that the back-calculation method can be easily implemented in order to avoid wind-up. To do so, the different integral actions associated with the same manipulated variable are collected. On the other hand, the proportional and derivative actions (PD) remain separated. For instance, the unsaturated control signal $\mathrm{u}_{1}$ would be calculated according to (53), where $e_{i}$ is the error signal. When the control signal is saturated while $u_{1}$ is different from $\mathrm{u}_{1 \_\mathrm{SAT}}$, the real process input $\mathrm{u}_{1 \_\mathrm{SAT}}$ works as tracking signal through the gain $\mathrm{K}_{\mathrm{R} 1}$. Then, the back-calculation method will make the integrator act in consequence to reduce this difference. Similar actions can be carried out on the other control signal $\mathrm{u}_{2}$.

$$
\begin{aligned}
& u_{1}(\mathrm{~s})=\left(K_{P 11}+\frac{K_{I 11}}{s}+\frac{K_{D 11} s}{T_{F 11} s+1}\right) e_{1}(\mathrm{~s})+\left(K_{P 12}+\frac{K_{I 12}}{s}+\frac{K_{D 12} s}{T_{F 12} s+1}\right) e_{2}(\mathrm{~s})+\frac{K_{R 1}\left(u_{1 S A T}(\mathrm{~s})-u_{1}(\mathrm{~s})\right)}{s}= \\
& =\left(K_{P 11}+\frac{K_{D 11} s}{T_{F 11} s+1}\right) e_{1}(\mathrm{~s})+\left(K_{P 12}+\frac{K_{D 12} s}{T_{F 12} s+1}\right) e_{2}(\mathrm{~s})+\frac{\left(K_{I 11} e_{1}(\mathrm{~s})+K_{I 12} e_{2}(\mathrm{~s})\right)+K_{R 1}\left(u_{1 S A T}(\mathrm{~s})-u_{1}(\mathrm{~s})\right)}{s}= \\
& =\mathrm{PD}_{11} e_{1}(\mathrm{~s})+\mathrm{PD}_{12} e_{2}(\mathrm{~s})+\frac{\left(K_{I 11} e_{1}(\mathrm{~s})+K_{I 12} e_{2}(\mathrm{~s})\right)+K_{R 1}\left(u_{1 S A T}(\mathrm{~s})-u_{1}(\mathrm{~s})\right)}{s}
\end{aligned}
$$




\section{Illustrative examples}

In this section, two simulation processes are considered to test the proposed methodology. In addition, its effectiveness is verified in a real quadruple tank plant. More simulation examples can be found in previous works (Garrido, Morilla, \& Vázquez, 2009; Morilla et al., 2008).

\subsection{Example 1: Depropanizer column}

The model of this process (Waller et al., 2003) is given by the $3 \times 3$ transfer function matrix in (54).

$$
\mathrm{G}_{\mathrm{D}}(\mathrm{s})=\left(\begin{array}{cccc}
\frac{-0.26978 e^{-27.5 s}}{97.5 s+1} & \frac{1.978 e^{-53.5 s}}{118.5 s+1} & \frac{0.07724 e^{-56 s}}{96 s+1} \\
\frac{0.4881 e^{-117 s}}{56 s+1} & \frac{-5.26 e^{-26.5 s}}{58.5 s+1} & \frac{0.19996 e^{-35 s}}{51 s+1} \\
\frac{0.6 e^{-16.5 s}}{40.5 s+1} & \frac{5.5 e^{-15.5 s}}{19.5 s+1} & \frac{-0.5 e^{-17 s}}{18 s+1}
\end{array}\right)
$$

Because of the time delays of (54), it is necessary to approximate $\left|G_{D}(s)\right|$ and the elements of the adjugate matrix $\operatorname{adj}_{\mathrm{D}}(\mathrm{s})$ into rational transfer functions plus time delay to apply the proposed methodology. These approximations are carried out by means of the method explained in section 3.1. The Nyquist diagrams and the singular values of the original frequency responses and their approximations are shown in Figures 7 and 8 for $\left|G_{D}(s)\right|$ and $\operatorname{adj}_{D}(s)$, respectively.
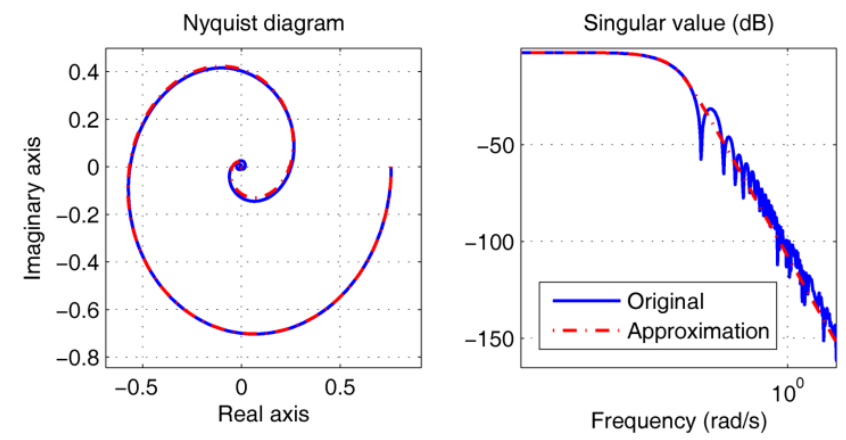
Figure 7. Nyquist diagram and singular value of the determinant of the process and its approximation in example 1.

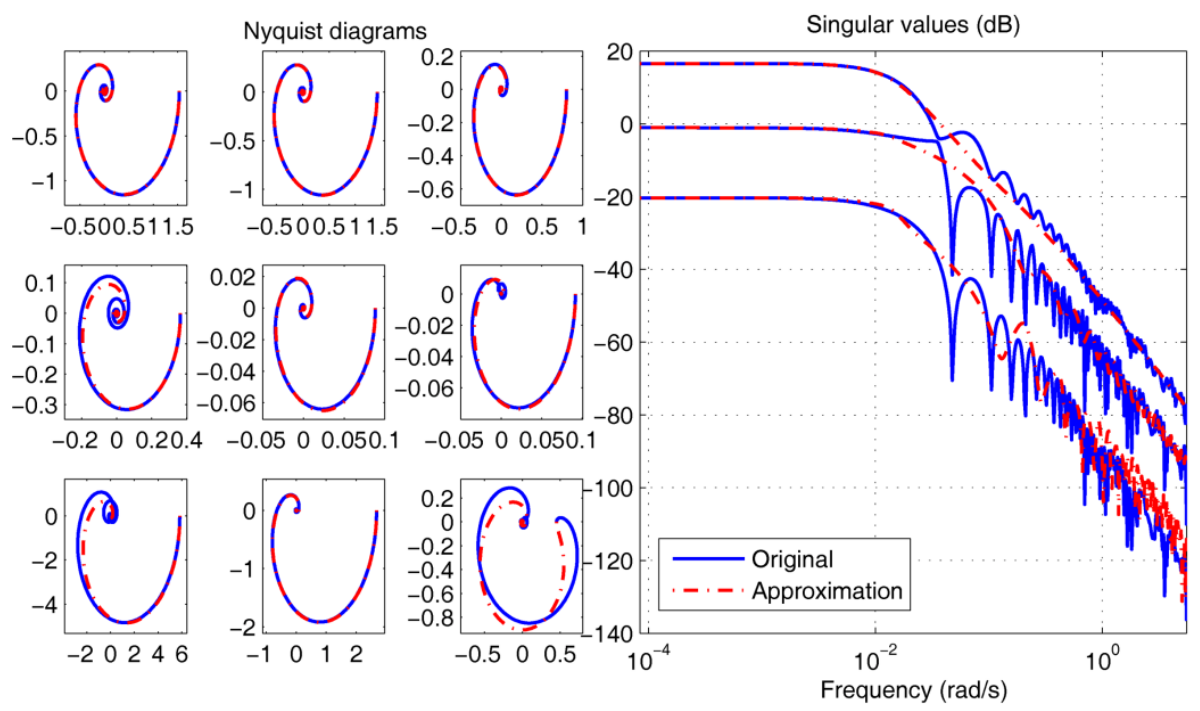

Figure 8. Nyquist diagrams and singular values of the process adjugate matrix and its approximation in example 1.

Specifically, the resultant approximated determinant is given by (55), a rational transfer function of relative degree 3 with a RHP zero at s=0.0118 and a time delay of $71 \mathrm{~s}$. The elements of $\operatorname{adjG}_{\mathrm{D}}(\mathrm{s})$ are approximated by (56). They are rational transfer functions with relative degree 2 , different time delays and no zeros at $\mathrm{s}=0.0118$.

$$
\begin{gathered}
\left|G_{D}(\mathrm{~s})\right|=\frac{4.2373 \cdot 10^{-6} \cdot(-\mathrm{s}+0.0118) e^{-71 s}}{(\mathrm{~s}+0.01721)(\mathrm{s}+0.008192)\left(\mathrm{s}^{2}+0.03088 \mathrm{~s}+0.0004686\right)} \\
\operatorname{adj}_{D}(\mathrm{~s})=\left[\begin{array}{ccc}
\frac{(12.67 \mathrm{~s}+1.53) e^{-43.5 s}}{3478 \mathrm{~s}^{3}+1300 \mathrm{~s}^{2}+84.43 s+1} & \frac{1.414 e^{-70.5 s}}{2073 s^{2}+130 s+1} & \frac{0.8018 e^{-82.5 s}}{6262 s^{2}+163.4 s+1} \\
\frac{(-5.872 \mathrm{~s}+0.364) e^{-51.5 s}}{10980 \mathrm{~s}^{3}+3785 s^{2}+109.1 s+1} & \frac{(1.428 \mathrm{~s}+0.08855) e^{-44.5 s}}{19220 \mathrm{~s}^{3}+2145 s^{2}+103.5 s+1} & \frac{0.09165 e^{-62.5 s}}{11950 s^{2}+190.6 s+1} \\
\frac{5.841 e^{-43 s}}{5217 s^{2}+119 s+1} & \frac{2.671 e^{-43 s}}{4163 s^{2}+147 s+1} & \frac{(37.65 \mathrm{~s}+0.4536) e^{-54 s}}{130200 \mathrm{~s}^{3}+6319 s^{2}+78.47 s+1}
\end{array}\right]
\end{gathered}
$$

Then, the desired diagonal open loop process $L_{D}(\mathrm{~s})=\operatorname{diag}\left\{l_{1}(\mathrm{~s}) I_{2}(s) l_{3}(\mathrm{~s})\right\}$ must be defined. The necessary dynamics in the three $\overline{l_{j}}(\mathrm{~s})$ elements to achieve realizability 
into the controllers of $K(\mathrm{~s})$ are calculated according to conditions (6), (7) and (8) for each column. Since $|G(s)|$ has a RHP cero, it is proposed to define the three desired open loop transfer functions $l_{j}(\mathrm{~s})$ according to the case 2 of Table 1 . Using a gain margin of 3 as performance specification in all the loops, the $k_{j}$ gains are calculated by means of (15) and (16), obtaining the desired open loop process transfer matrix $L_{D}(\mathrm{~s})$ in (57).

$$
L_{D}(\mathrm{~s})=\operatorname{diag}\left\{\frac{0.003(-\mathrm{s}+0.0118) e^{-28 \mathrm{~s}}}{(\mathrm{~s}+0.0118) \mathrm{s}} \frac{0.003(-\mathrm{s}+0.0118) e^{-28 \mathrm{~s}}}{(\mathrm{~s}+0.0118) \mathrm{s}} \frac{0.0033(-\mathrm{s}+0.0118) e^{-17 \mathrm{~s}}}{(\mathrm{~s}+0.0118) \mathrm{s}}\right\}
$$

The elements of the centralized control by decoupling $\mathrm{K}(\mathrm{s})$ are obtained according to (3). Then, they are approximated to the parallel PID structure in (49) using the reduction method described in Section 3.2. The parameters of the resulting multivariable PID control are shown in (58). Figure 9 shows the singular values of the original controller $K(\mathrm{~s})$ and its PID approximation.

$$
\begin{aligned}
K_{P} & =\left(\begin{array}{ccc}
0.485 & 0.092 & -0.019 \\
0.02 & 0.024 & 0.002 \\
0.994 & 0.155 & 0.33
\end{array}\right) K_{D}=\left(\begin{array}{ccc}
0 & 0 & 0 \\
0 & 0.43 & 0 \\
66.7 & 13.9 & -3.39
\end{array}\right) \\
K_{I} & =\left(\begin{array}{ccc}
0.613 & 0.567 & 0.351 \\
0.146 & 0.035 & 0.04 \\
2.34 & 1.07 & 0.2
\end{array}\right) / 100 T_{F}=\left(\begin{array}{ccc}
0 & 0 & 0 \\
0 & 6.26 & 0 \\
132.7 & 44.7 & 11.7
\end{array}\right)
\end{aligned}
$$

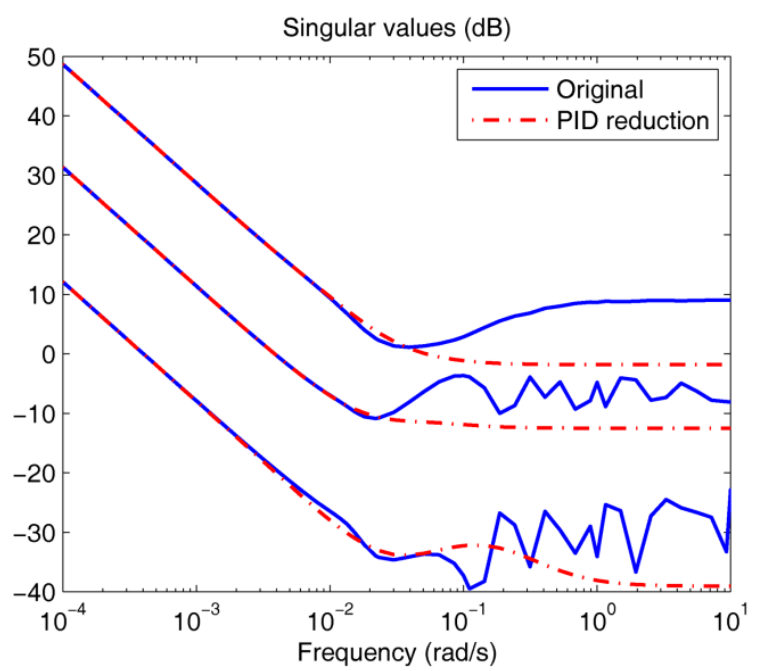


Figure 9. Singular values of the ideal controller $\mathrm{K}(\mathrm{s})$ and its multivariable PID approximation in example 1.

Figure 10 shows the closed loop system response of the proposed methodology in comparison with those of the centralized non-PID control of Wang in (Wang, 2003) and a multiloop PID controller. The multiloop PID controller is tuned according to Lee's method (Lee et al., 2004) to achieve similar performance: a gain margin of 3 in each loop. The chosen pairing is the paring recommended by the relative gain array (RGA). The PID parameters are given in (59). There are unit step changes at $t=0 \mathrm{~s}$ in the first reference, at $t=2000 \mathrm{~s}$ in the second reference, and at $\mathrm{t}=4000 \mathrm{~s}$ in the third one; at $\mathrm{t}=6000 \mathrm{~s}$, there is a $0.1 \mathrm{step}$ in all process inputs at the same time as input disturbance. The IAE of each loop is obtained as performance indices and collected in Table 2.

$$
\begin{aligned}
K_{P} & =\left(\begin{array}{ccc}
0 & 0 & 0.32 \\
0.25 & 0 & 0 \\
0 & 1.14 & 0
\end{array}\right) K_{D}=\left(\begin{array}{ccc}
0 & 0 & 0.17 \\
1.2 & 0 & 0 \\
0 & 2.32 & 0
\end{array}\right) \\
K_{I} & =\left(\begin{array}{ccc}
0 & 0 & 0.49 \\
0.19 & 0 & 0 \\
0 & 1.5 & 0
\end{array}\right) / 100 T_{F}=\left(\begin{array}{ccc}
0 & 0 & 0.05 \\
0.48 & 0 & 0 \\
0 & 0.2 & 0
\end{array}\right)
\end{aligned}
$$
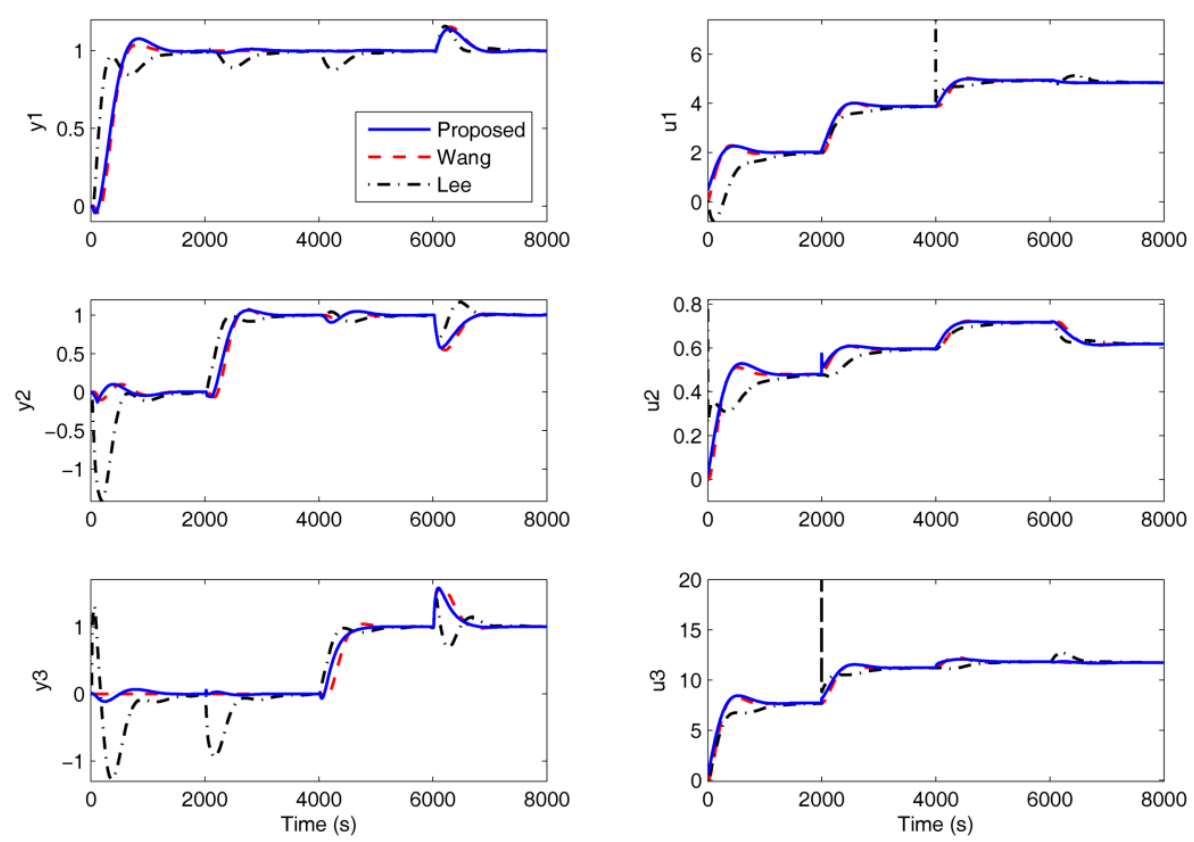
Figure 10. Outputs and control signals of the step response in example 1.

The response of the proposed method is quite similar to that of Wang's controller; however, the proposed methods produce smaller IAE. In addition, the complexity of the proposed multivariable PID control is much simpler than that of Wang's control. Both perform better than the multiloop PID control that has very important interactions in outputs $\mathrm{y}_{2}$ and $\mathrm{y}_{3}$.

Table 2. IAE values and robustness indices for each method in example 1.

\begin{tabular}{lccccc}
\hline Method & $\mathrm{IAE}_{1}$ & $\mathbf{I A E}_{2}$ & $\mathrm{IAE}_{3}$ & $\boldsymbol{\mu}_{\mathrm{RS}}$ & $\boldsymbol{\mu}_{\mathrm{RP}}$ \\
\hline Proposed & 473 & 643 & 557 & 0.16 & 1.04 \\
Wang & 474 & 708 & 612 & 0.17 & 1.19 \\
$\begin{array}{l}\text { Lee } \\
\left(\lambda_{1}=200,\right. \\
\lambda_{2}=200, \\
\left.\lambda_{3}=200\right)\end{array}$ & 432 & 911 & 1413 & 0.33 & 2.02 \\
\hline
\end{tabular}

To evaluate the robustness of the proposed controller, a $\mu$-analysis is performed in the presence of diagonal multiplicative input uncertainty using the weights in (60). The weight $w_{I}(\mathrm{~s})$ can be interpreted as the process inputs increase by up to $200 \%$ uncertainty at high frequencies and by almost $15 \%$ uncertainty in the low frequency range. The performance weight $w_{P}(\mathrm{~s})$ specifies integral action and a maximum peak for $\bar{\sigma}(\mathrm{S})$ of 2.75 . The SSV for robust stability (RS) and robust performance (RP) for the different controllers under conditions (28) and (29) are shown in Figure 11. The peak values are shown in Table 2. The proposed controller obtains the smallest ones. The RS is smaller than one for all frequencies, indicating that the systems will remain stable in spite of an uncertainty of $15 \%$ on each process input. The multiloop PID controller has a good RS; however, it shows the worst robust performance that is due to the strong interactions in the second and third loops. 


$$
\begin{aligned}
& W_{I}(\mathrm{~s})=W_{I}(\mathrm{~s})=\frac{(0.009 \mathrm{~s}+0.15)}{0.0045 s+1} \cdot I \\
& W_{P}(\mathrm{~s})=W_{P}(\mathrm{~s})=\frac{(\mathrm{s} / 2.75+0.00075)}{s} \cdot I
\end{aligned}
$$
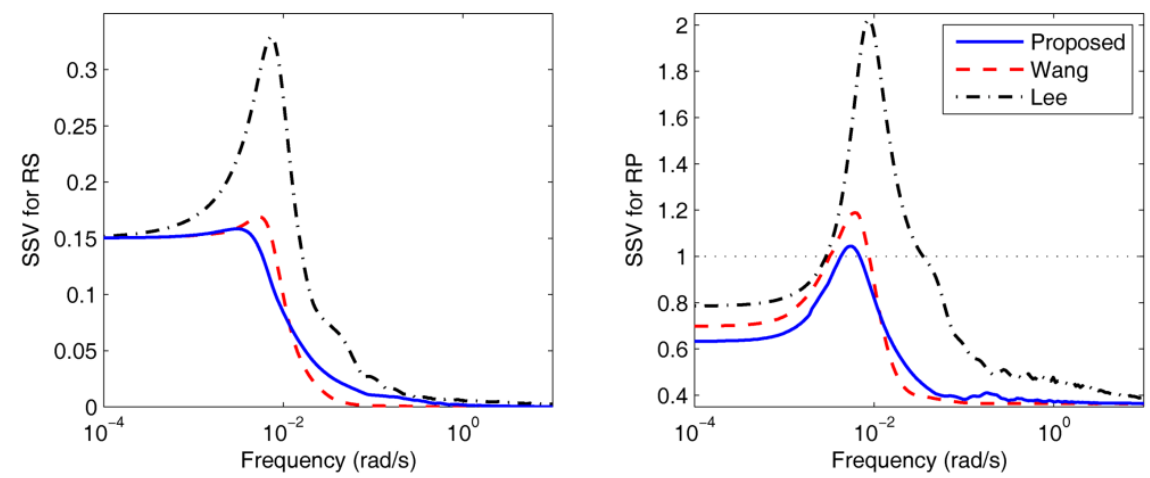

Figure 11. SSV for RS and RP in example 1.

\subsection{Example 2: Boiler process}

The model of this process is given by the $2 \times 2$ transfer matrix in (61). This model is obtained from a linearization of the boiler control problem proposed in (Morilla, 2012). It has been chosen as an example in order to show the cases 1 and 3 of Table 1.

$$
G_{B}(\mathrm{~s})=\left(\begin{array}{cc}
\frac{0.308}{28.96 \mathrm{~s}+1} & \frac{-0.159}{183.7 \mathrm{~s}+1} \\
\frac{-0.0055872 \cdot(-166.9 \mathrm{~s}+1)}{\mathrm{s}(26.38 \mathrm{~s}+1)} & \frac{0.010645}{\mathrm{~s}}
\end{array}\right)
$$

The determinant and adjugate matrix of the process do not need to be approximated since there are no time delays. In addition, they do not have RHP zeros. The dynamics associated to the first column of the inverse of G(s) are cancellable. Thus, $l_{1}(\mathrm{~s})$ is specified according to the case 1 of Table 1 without delay. Using a closed loop time constant of $20 \mathrm{~s}$ as specification, it is obtained that $k_{1}=0.05$. The elements of the second column of the inverse of $\mathrm{G}(\mathrm{s})$ have a zero at $\mathrm{s}=0$ and therefore, $l_{2}(\mathrm{~s})$ can be defined according to the case 3 of Table 1 without time delay. Using a critical damping and a natural frequency of $0.0628 \mathrm{rad} / \mathrm{s}$ as closed loop specifications, the $l_{2}(\mathrm{~s})$ 
parameters $\left(k_{2}=0.1257\right.$ and $\left.z=0.0314\right)$ are obtained from (22). Consequently, the desired open loop process is given by

$$
L_{B}(\mathrm{~s})=\operatorname{diag}\left\{\frac{0.05}{\mathrm{~s}} \frac{0.1257(\mathrm{~s}+0.0314)}{\mathrm{s}^{2}}\right\}
$$

Then, the ideal controller elements are obtained from (3) resulting third order elements. Therefore, they are approximated to PID structure using the proposed method in Section 3. The parameters of the PID controllers are shown in (63).

$$
\begin{aligned}
& K_{P}=\left(\begin{array}{ll}
-22.3 & -66.2 \\
-34.3 & -49.6
\end{array}\right) \quad K_{I}=\left(\begin{array}{ll}
0.22 & 0.26 \\
0.11 & 0.51
\end{array}\right) \\
& K_{D}=\left(\begin{array}{ll}
0.81 & 2.09 \\
0.71 & 1.84
\end{array}\right) \quad T_{F}=\left(\begin{array}{ll}
312.4 & 312.4 \\
315.5 & 312.4
\end{array}\right)
\end{aligned}
$$

Figure 12 shows the closed loop system response of the proposed controller in comparison with those obtained with the reference decentralized PI controller in (Morilla, 2012) and the multivariable PI control in (Ochi \& Yokoyama, 2012). There is a unit step change in the first reference at $t=0 \mathrm{~s}$, and at $t=1000 \mathrm{~s}$, in the second one. At $t=2000 \mathrm{~s}$, there is a 2 step in both process inputs as load disturbances. The IAE indices are shown in Table 3.
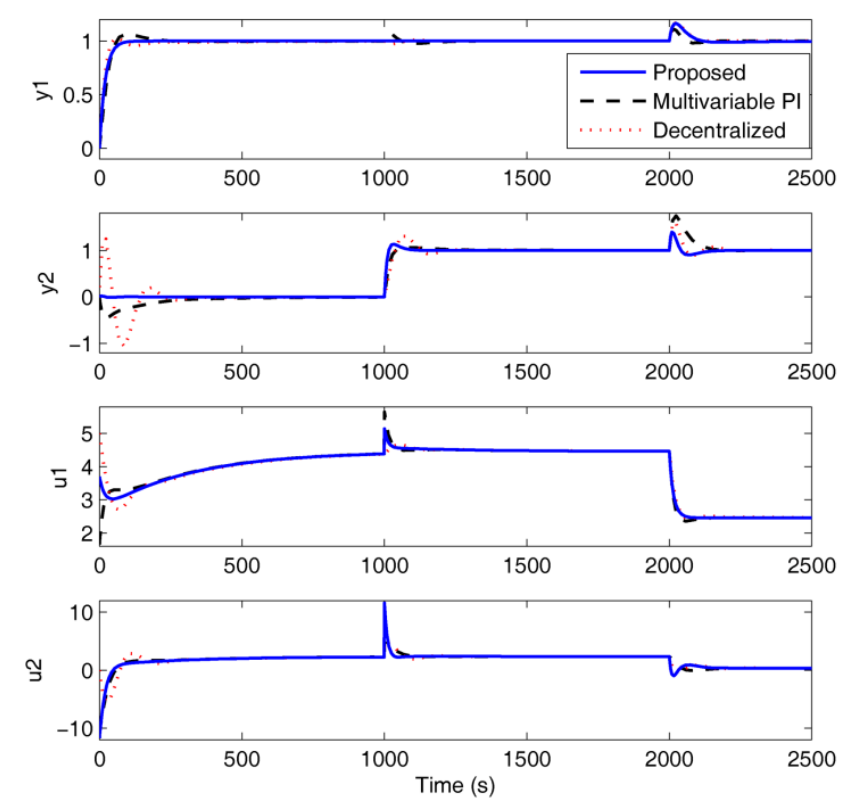
Figure 12. Outputs and control signals of the step response in example 2.

The proposed control achieves the best response with the smallest IAE values and almost perfect decoupling. The multivariable PI control of Ochi and the decentralized control present non-negligible interactions in the second loop.

Table 3. IAE values and robustness indices for each method in example 2.

\begin{tabular}{lcccc}
\hline Method & $\mathbf{I A E}_{\mathbf{1}}$ & $\mathbf{I A E}_{\mathbf{2}}$ & $\boldsymbol{\mu}_{\mathrm{RS}}$ & $\boldsymbol{\mu}_{\mathrm{RP}}$ \\
\hline Proposed & 31.8 & 28.7 & 0.35 & 1 \\
$\begin{array}{l}\text { Multivariable } \\
\text { PI }\end{array}$ & 40.2 & 145 & 0.37 & 2.6 \\
Decentralized & 35.2 & 191 & 0.54 & 4.36 \\
\hline
\end{tabular}

To investigate the robustness of the three controllers, a $\mu$-analysis similar to the previous example is performed. The selected weights are given by (64). The SSV for RS and RP for the different controllers are shown in Figure 13. The RS is satisfied by all of them; however, the smallest values are obtained by the proposed control. The peak values are collected in Table 3. The RP condition (30) is only satisfied by the proposed control. The other ones show bad robust performance at low and medium frequencies.

$$
\begin{aligned}
& W_{I}(\mathrm{~s})=W_{I}(\mathrm{~s})=\frac{(\mathrm{s}+0.3)}{\mathrm{s}+1} \cdot I \\
& W_{P}(\mathrm{~s})=W_{P}(\mathrm{~s})=\frac{(\mathrm{s} / 1.5+0.03)}{s} \cdot I
\end{aligned}
$$
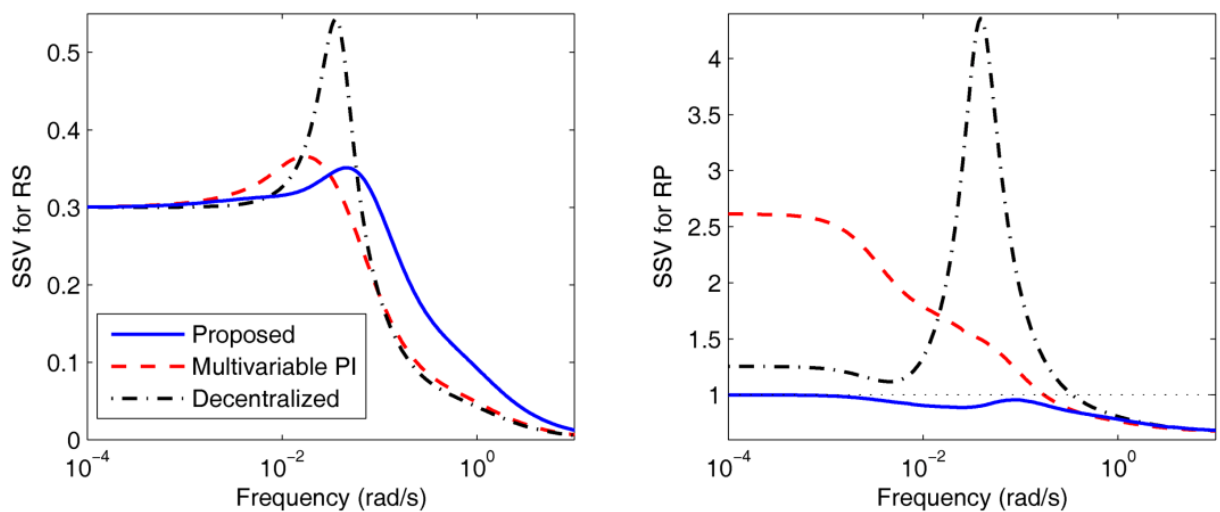
Figure 13. SSV for RS and RP in example 2.

Next, a simulation is carried out with input constraints in order to illustrate the windup problem and check the anti-windup (AW) scheme of Figure 6. The first process inputs is limited in the range of $[1,5]$, and the second one, in the range of $[0,5]$. At $t=0$ $\mathrm{s}$, there is a unit step change in the second reference, and then, at $t=1000 \mathrm{~s}$, there is a similar step change in the first one. The proposed controller is tested assuming two cases: without anti-windup mechanism and using the proposed anti-windup scheme. Simulation results are shown in Figure 14. After the first step in the second reference, the first control signal $u_{1}$ should be out of range to track this reference. However, the lower limit of $u_{1}$ is reached and the references cannot be achieved. In the first case, without anti-windup mechanism, this fact provokes windup in this signal and after the step in the first reference, at $t=1000 \mathrm{~s}, u_{1}$ does not change until $250 \mathrm{~s}$ later. Consequently, the time to reach the new references is very late. This response is improved significantly over the implementation of the anti-windup scheme of Figure 6. The windup effect has been avoided, the control signal $u_{1}$ reacts very quickly after the second step, and all references are reached sooner.
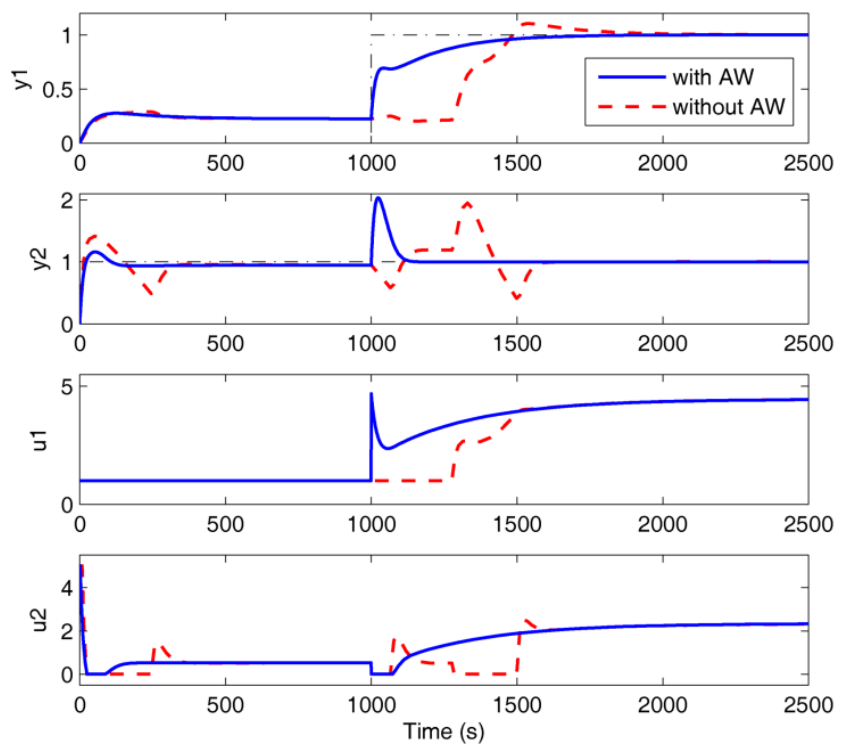
Figure 14. Outputs and control signals of the step response of Example 2 with input constraints.

\subsection{Example 3: Experimental quadruple tank system}

The experimental process is a quadruple tank plant (Johansson, 2000) in the lab of the Computer Science Department of the University of Cordoba. The outputs are the level of the lower tanks, which are between 0 and $35 \mathrm{~cm}$; the inputs are the flow references of the secondary control loops that regulate the operation of the pumps, which are between 0 and $200 \mathrm{~cm}^{3} / \mathrm{s}$. The system was configured to show interaction problems with a multivariable RHP zero. The process was identified around the operation point $h=[17$ 18] $\mathrm{cm}$ and $\mathrm{u}=[135135] \mathrm{cm}^{3} / \mathrm{s}$. The resultant model is given by (65). It has an RGA of -0.21 and a multivariable RHP zero at $s=1 / 164.67$.

$$
\mathrm{G}_{\mathrm{T}}(\mathrm{s})=\left(\begin{array}{cc}
\frac{0.175}{191.5 s+1} & \frac{0.402}{(170.5 s+1)(260.7 s+1)} \\
\frac{0.385}{(165 s+1)(240.4 s+1)} & \frac{0.154}{178.6 s+1}
\end{array}\right)
$$

Because of this multivariable RHP zero, which needs to be included in the open loop process, the open loop transfer function $\mathrm{l}_{1}(\mathrm{~s})$ and $\mathrm{l}_{2}(\mathrm{~s})$ can be defined according to case 2 of Table 1. Since there are no time delays, the closed loop transfer functions are given by (17). Using a damping factor equal to the unity as desired specification in loops, the corresponding gains $\mathrm{k}_{1}$ and $\mathrm{k}_{2}$ can be calculated by means of (18). As a result, the desired open loop transfer functions are defined by

$$
l_{1}(\mathrm{~s})=l_{2}(\mathrm{~s})=\frac{1.042 \cdot 10^{-3}(-\mathrm{s}+1 / 164.67)}{s(\mathrm{~s}+1 / 164.67)}
$$

After that, the ideal controller elements are obtained from (3) or (27). Then, these elements are reduced to PI structure using the proposed approximation method of 
Section 3 in order to achieve the proposed multivariable PI control (MV-PI). The parameters of the resulting PI controllers are shown in Table 4.

Table 4. PI parameters for example 3.

\begin{tabular}{lll}
\hline \multirow{2}{*}{ Method } & \multicolumn{3}{l}{ PI parameters } \\
\cline { 2 - 3 }$K_{P}$ & \multicolumn{1}{l}{$T_{I}$} \\
\hline Proposed & $\left(\begin{array}{cc}-0.537 & 0.572 \\
0.629 & -0.591\end{array}\right)$ & $\left(\begin{array}{cc}427.4 & 174.2 \\
200 & 413.9\end{array}\right)$ \\
MV-PI & $\left(\begin{array}{cc}0 & 420 \\
432 & 0\end{array}\right)$ \\
Decentralized & $\left.\begin{array}{cc}0 & 3.96 \\
4.92 & 0\end{array}\right)$ & $\left(\mathrm{y}_{1}-\mathrm{u}_{2}\right),\left(\mathrm{y}_{2}-\mathrm{u}_{1}\right)$ \\
\hline
\end{tabular}

Figure 15 shows the resultant response of the proposed controllers for a step of 4 $\mathrm{cm}$ in both references. For comparison, the response of a decentralized PI controller is also shown. This controller is tuned according to the iterative method in (Vázquez et al., 1999) using a phase margin of $60^{\circ}$ as specification in both loops. According to the RGA, the chosen pairing is $\mathrm{y}_{1}-\mathrm{u}_{2}$ and $\mathrm{y}_{2}-\mathrm{u}_{1}$. The PI parameters are listed in Table 4.
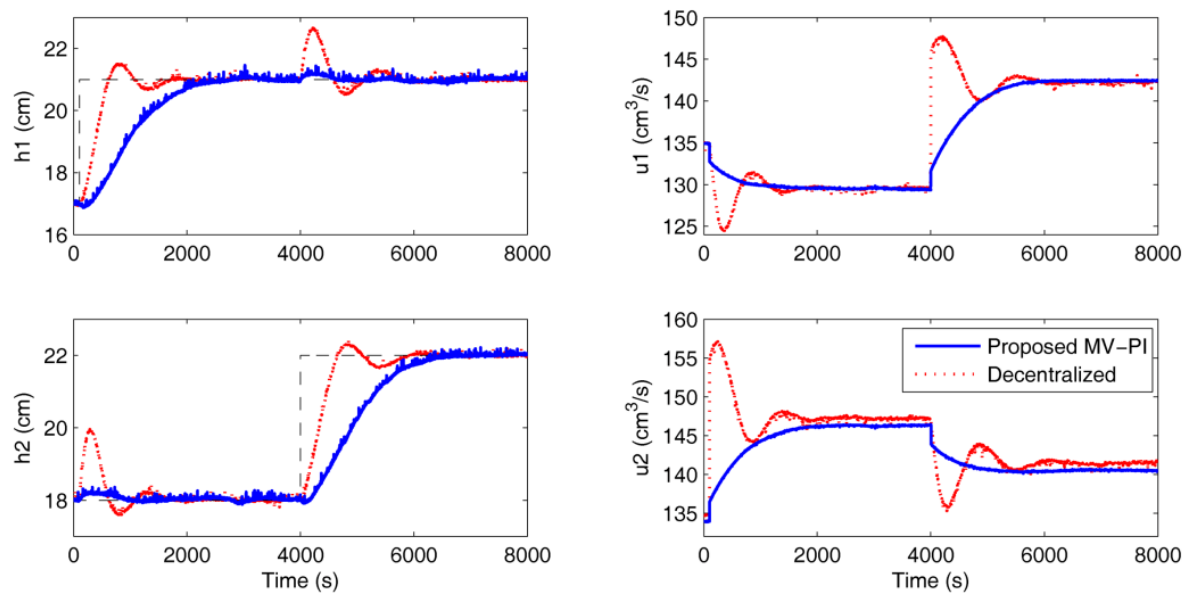

Figure 15. Outputs and control signals of the step response of the quadruple tank system.

With the proposed control, perfect decoupling is almost achieved with some little interactions, while the decentralized control presents important couplings in both loops. However, the improved decoupling performance of the proposed methodology is obtained at the expensive of closed loop bandwidth because the RHP zero imposes an 
upper limit on this bandwidth. The decentralized control reaches the references faster; nevertheless, its control signals are much rougher than those of the proposed method.

\section{Conclusions}

A new methodology of multivariable PID controllers based on decoupling control is developed in this work. The formulation is presented for general $n \times n$ systems with time delays. It consists of an ideal decoupling control with integral action which is designed to minimize interactions and to obtain zero error in steady state. This control depends on the desired open loop processes that are defined according to the realizability conditions and the desired performance specifications. These realizability conditions are stated and furthermore, three common cases to achieve performance specifications from simple open loop transfer functions are proposed. Then, the ideal controller elements are approximated to parallel PID structure. The parameter $\mathrm{k}_{\mathrm{j}}$, which is common by column in the control transfer matrix, can be used as a degree of freedom in order to modify the performance of the corresponding loop without changing the other controller elements and without almost affecting the performance of the other loops.

Several design considerations, such as the approximation method to PID controller, have been discussed and proposed. Additionally, anti-windup scheme for the multivariable PID controllers has been proposed from a practical point of view. This scheme, which can be implemented in distributed control systems, has been tested in simulation.

The method has been illustrated with several simulation examples. Comparisons with other methods have demonstrated that the proposed methodology achieves similar or better performance. Additionally, an experimental quadruple tank system was used to verify the effectiveness of these methodologies. 


\section{Acknowledgements}

This work was supported by the Autonomous Government of Andalusia (Spain) under the Excellence Project P10-TEP-6056; and the Spanish Ministry of Economy and Competitiveness under Grant DPI2012-37580-C02-01.

\section{References}

Alcántara, S., Vilanova, R., \& Pedret, C. (2013). PID control in terms of robustness/performance and servo/regulator trade-offs: A unifying approach to balanced autotuning. J. Process Control, 23(4), 527-542.

Áström, K., \& Hägglund, T. (2006). Advanced PID control. Research Triangle Park, North Carolina: ISA-The Instrumentation, Systems, and Automation Society.

Cai, W. J., Ni, W., He, M. J., \& Ni, C. Y. (2008). Normalized decoupling - a new approach for MIMO process control system design. Ind. Eng. Chem. Res., 47(19), 7347-7356.

Chien, I. L., Huang, H. P., \& Yang, J. C. (1999). A simple multiloop tuning method for PID controllers with no proportional kick. Ind. Eng. Chem. Res., 38(4), 1456-1468.

Darby, M. L., \& Nikolaou, M. (2012). MPC: Currentpracticeandchallenges. Control Eng. Pract., 20(4), 328-342.

Garrido, J., Morilla, F., \& Vázquez, F. (2009). Centralized PID Control by Decoupling of a BoilerTurbine Unit. Paper presented at the European Control Conference Budapest.

Garrido, J., Vázquez, F., \& Morilla, F. (2011). An extended approach of inverted decoupling. J. Process Control, 21(1), 55-68.

Garrido, J., Vázquez, F., \& Morilla, F. (2012). Centralized multivariable control by simplified decoupling. J. Process Control, 22(6), 1044-1066.

Garrido, J., Vázquez, F., \& Morilla, F. (2013). Centralized Inverted Decoupling Control. Ind. Eng. Chem. Res., 52(23), 7854-7866.

Garrido, J., Vázquez, F., Morilla, F., \& Hägglund, T. (2011). Practical advantages of inverted decoupling. Proc. Inst. Mech. Eng. I J. Syst. Control Eng., 225(7), 977-992.

Goodwin, G. C., Graebe, S. F., \& Salgado, M. E. (2001). Control System Design. New Jersey, USA: Prentice-Hall.

Jevtovic, B. T., \& Matausek, M. R. (2010). PID controller design of TITO system based on ideal decoupler. J. Process Control, 20(7), 869-876.

Johansson, K. H. (2000). The quadruple-tank process: A multivariable laboratory process with an adjustable zero. IEEE Trans Control Syst Technol., 8(3), 456-465.

Lee, M., Lee, K., Kim, C., \& Lee, J. (2004). Analytical design of multiloop PID controllers for desired closed-loop responses. AIChE J., 50(7), 1631-1635.

Lieslehto, J. (1996). MIMO controller design using SISO controller design methods. Paper presented at the Proceeding of the 13th IFAC World Congress, San Francisco, USA.

Liu, T., Zhang, W., \& Gao, F. (2007). Analytical decoupling control strategy using a unity feedback control structure for MIMO processes with time delays. J. Process Control, 17(2), 173-186.

Morilla, F. (2012). Benchmark for PID control based on the boiler control problem. Paper presented at the IFAC Conference on Advances in PID Control PID’12, Brescia, Italy.

Morilla, F., Garrido, J., \& Vázquez, F. (2013). Control Multivariable por Desacoplo. Rev. Iberoam. Autom. Inform. Ind., 10(1), 3-17.

Morilla, F., Vázquez, F., \& Garrido, J. (2008). Centralized PID control by decoupling for TITO processes. Paper presented at the Proceedings of the 13th IEEE International Conference on Emerging Technologies and Factory Automation, Hamburg, Germany.

Ochi, Y., \& Yokoyama, N. (2012). PID Controller Design for MIMO Systems by Applying Balanced Truncation to Integral-Type Optimal Servomechanism. Paper presented at the IFAC Conference on Advances in PID Control PID’12, Brescia, Italy.

Pintelon, R., Guillaume, P., Rolain, Y., Schoukens, J., \& Van hamme, H. (1994). Parametric identification of transfer functions in the frequency domain - A survey. IEEE Trans. Autom. Control, 39(11), 2245-2260. 
Rajapandiyan, C., \& Chidambaram, M. (2012). Controller design for MIMO processes based on simple decoupled equivalent transfer functions and simplified decoupler. Ind. Eng. Chem. Res., 51(38), 12398-12410.

Rivera, D. E., Morari, M., \& Skogestad, S. (1986). Internal model control. 4. PID controller design. Ind. Eng. Chem. Process Des. Dev., 25(1), 252-265.

Saeki, M. (2006). Fixed structure PID controller design for standardHळ control problem. Automatica, 42(1), 93-100.

Shen, Y., Sun, Y., \& Li, S. (2012). Adjoint transfer matrix based decoupling control for multivariable processes. Ind. Eng. Chem. Res., 51(50), 16419-16426.

Shinskey, F. G. (1979). Process Control Systems: Application, Design and Adjustment (2 ed.). New York: McGraw-Hill.

Skogestad, S., \& Postlethwaite, I. (2005). Multivariable Feedback Control: Analysis and Design (2 ed.). Chichester, England: John Wiley \& sons.

Vázquez, F., Morilla, F., \& Dormido, S. (1999). An iterative method for tuning decentralized PID controllers. Paper presented at the Proceedings of the 14th IFAC World Congress, Beijing, China.

Waller, M., Waller, J. B., \& Waller, K. V. (2003). Decoupling Revisited. Ind. Eng. Chem. Res., 42(20), $4575-4577$.

Wang, Q. G. (2003). Decoupling Control. Berlin-Heidelberg: Springer-Verlag.

Wang, Q. G., Zhang, Y., \& Chiu, M. S. (2002). Decoupling internal model control for multivariable systems with multiple time delays. Chem. Eng. Sci., 57(1), 115-124.

Wang, Q. G., Zhang, Y., \& Chiu, M. S. (2003). Non-interacting control design for multivariable industrial processes. J. Process Control, 13(3), 253-265.

Wang, Q. G., Zou, B., Lee, T. H., \& Bi, Q. (1997). Auto-tuning of multivariable PID controllers from decentralized relay feedback. Automatica, 33(3), 319-330.

Xiong, Q., \& Cai, W.-J. (2006). Effective transfer function method for decentralized control system design of multi-input multi-output processes. J. Process Control, 16(8), 773-784. doi: 10.1016/j.jprocont.2006.04.001

Xiong, Q., Cai, W. J., \& He, M. J. (2007). Equivalent transfer function method for PI/PID controller design of MIMO processes. J. Process Control, 17(8), 665-673.

Zhang, W., Chen, L., \& Ou, L. (2006). Algebraic Solution to H2 Control Problems. II. The Multivariable Decoupling Case. Ind. Eng. Chem. Res., 45(21), 7163-7176.

Zheng, F., Wang, Q. W., \& Lee, T. H. (2002). On the design of multivariable PID controllers via LMI approach. Automatica, 38(3), 517-526.

Zhu, Y., Patwardhan, R., Wagner, S. B., \& Zhao, J. (2013). Toward a low cost and high performance MPC: The role of system identification. Comput. Chem. Eng., 51(5), 124-135. 